\title{
UNIVERSAL MODELS FOR LORENZ MAPS
}

\author{
Marco Martens ${ }^{1}$ and Welington de Melo $^{2}$
}

September 23, 1996

\begin{abstract}
.
The existence of smooth families of Lorenz maps exhibiting all possible dynamical behavior is established and the structure of the parameter space of these families is described.
\end{abstract}

\footnotetext{
${ }^{1}$ Institute of Mathematical Sciences, SUNY at Stony Brook, Stony Brook, NY 11794-3651.

2IMPA, Estrada Dona Castorina 110, 22460-320 Rio de Janeiro, Brazil. 


\section{Introduction}

The aim of this paper is to exhibit some parameterized families of Lorenz flows that are topologically universal in the sense that given any geometric Lorenz flow, its dynamics is essentially the same as the dynamics of some element of the family. Thus, these families plays, in the context of Lorenz flows, the same role as the quadratic family in the context of unimodal interval maps.

Lorenz in $[\mathrm{L}]$ showed numerically the existence of some flows in three dimension that have complicated recurrent behavior. What we now call a Lorenz flow has a singularity of saddle type with a one dimensional unstable manifold and an infinite set of hyperbolic periodic orbits, whose closure contains the saddle point. More specifically, the closure of this set of periodic orbits is in general the global attractor of the flow.

To analyze the dynamics of such a flow we take a two dimensional transversal section intersecting the local stable manifold in a line $l$ and we look at the first return map to $S$. This map is not defined in the line $l$ and in fact exhibits a discontinuity at $l$ because orbits near $l$ in opposite sides follows different branches of the unstable manifold. To describe the dynamics of such a flow, Guckenheimer and Williams added a new hypothesis: the existence of a one dimension foliation in $S$ that is invariant by the first return map, has $l$ as a leaf and is such that points in the same leaf are exponentially contracted under iteration by the first return map. A Lorenz flow with this extra structure we call a geometric Lorenz flow. Because of the exponential contraction on the leaves of the foliation, the dynamics of such a flow can be described by the action of the first return map on the space of leaves of the stable foliation. This space of leaves is an interval and the induced map has a unique discontinuity at the point corresponding to $l$. Such an interval map we call a Lorenz map. More precisely,

Definition 1.1. Let $P<0<Q$. A Lorenz map from $[P, Q]$ to $[P, Q]$ is a pair $\left(f_{-}, f_{+}\right)$ where

1) $f_{-}:[P, 0] \rightarrow[P, Q]$ and $f_{+}:[0, Q] \rightarrow[P, Q]$ are continuous and strictly increasing maps.

2) $f(P)=P$ and $f(Q)=Q$.

3) Given $\rho>0$, we will say that $f$ is a $C^{r}$ of exponent $\rho$ if we can write

$$
f_{-}(x)=\tilde{f}_{-}\left(x^{\rho}\right) \text { and } f_{+}(x)=\tilde{f}_{+}\left(x^{\rho}\right)
$$

where $\tilde{f}_{-}$and $\tilde{f}_{+}$are $C^{r}$ diffeomorphisms defined on appropriate closed intervals. Notation: This Lorenz map is denoted by $\left(P, Q, f_{-}, f_{+}\right)$.

Notice that if $r \geq 1$ then the triple $\left\{\rho, \tilde{f}_{-}, \tilde{f}_{+}\right\}$is uniquely determined by $f$. If the map is associated to a Lorenz vector field then the exponent $\rho$ is precisely the absolute value of the ratio between the unstable eigenvalue and the weak stable eigenvalue at the saddle point. 
A Lorenz map is non-trivial iff $f([P, 0]) \supset[P, 0]$ and $f([0, Q]) \supset[0, Q]$. Otherwise $f$ is trivial, any orbit of such a map is asymptotic to a fixed point.

Guckenheimer and Williams proved in $[\mathrm{GW}]$ that there exists an open set of vector fields in three space that have a structure of geometric Lorenz flow with smooth associated Lorenz maps. In fact they only considered the situation where the exponent is smaller than one and the map expanding with derivative everywhere bigger than $\sqrt{2}$. However we can use the same arguments to construct open sets of vector fields having Lorenz maps with exponent bigger than one. As we will see soon, the Lorenz maps of exponent bigger than one presents a much bigger variety of dynamical behavior due to the interplay of contractions and expansions. Compare this with the unimodal situation: the quadratic family exhibits more types of combinatorics than the expanding tent family. Before stating our results we need to discuss some combinatorial aspects of Lorenz maps.

A branch of $f^{n}$ is a maximal closed interval $J$ such that $f^{n}$ is a diffeomorphism in the interior of $I$. So an end point of $J$ is either $P$, or $Q$ or a point in the backward orbit of 0 . To each branch $J$ of $f^{n}$ we can associate a word $\alpha=\left(\alpha_{0}, \alpha_{1}, \ldots, \alpha_{n-1}\right)$ where $\alpha_{i} \in\{[P, 0],[0, Q]\}$ and $f^{i}(J) \subset \alpha_{i}$. It is clear that given a word of length $n$, there exists at most one branch of $f^{n}$ associated to it. The combinatorics of all possible words are determined by the kneading invariants of $f, K_{-}(f)$ and $K_{+}(f)$ defined as follows: the first $n$ symbols of $K_{-}(f)$ are the symbols of the branch of $f^{n+1}$ adjacent to 0 that is contained in $[P, 0]$. Similarly for $K_{+}(f)$. There are many papers describing the combinatorics of Lorenz maps, see, for example, $[\mathrm{P}]$ and specially $[\mathrm{HS}]$ where all possible kneading invariants of Lorenz maps are characterized.

The intersection of all branches that contains a given point is either a point or a closed interval. If such an intersection is an interval, it is called a homterval of $f$. A critical homterval is a homterval that has 0 as an endpoint. So there are at most two critical homtervals. The image of a homterval is always contained in another homterval and, if the homterval is not critical, it is onto. An orbit of homterval is a sequence $J_{0}, J^{1}, \ldots$ of homtervals such that $f\left(J^{n}\right) \subset J^{n+1}$. There are three types of orbits of homtervals:

1) $J^{0}$ is a wandering interval if its orbit contains infinitely many intervals;

2) it is periodic of period $n$ if $J^{n}=J_{0}$ and $J^{i} \neq J^{0}$ if $0<i<n$;

3 ) it is eventually periodic if it is not periodic but $J^{i}$ is periodic for some $i$.

Definition 1.2. We say that $f$ is a simple Lorenz map if $f$ has no homterval.

Definition 1.3. A maximal semi conjugacy from a Lorenz map $f$ to a Lorenz map $\hat{f}$ is a continuous, monotone, surjective map $h$ such that:

1) $\hat{f} \circ h=h \circ f$;

2) the inverse image of each point is either a point or a homterval or an interval whose points are all asymptotic to periodic points.

In section 6 we will discuss this notion of semi conjugacy. Roughly speaking, a maximal semi conjugacy collapses as much as possible without destroying essential parts of the 
dynamics. If $f$ has at most one critical homterval then a maximal semi conjugacy collapses only homtervals.

Definition 1.4. The Lorenz maps $f_{1}$ and $f_{2}$ are said to be essentially conjugated iff there exists a simple Lorenz map $\hat{f}$ and nice semi conjugacies $h_{1}$, from $f_{1}$ to $\hat{f}$, and $h_{2}$ from $f_{2}$ to $\hat{f}$.

Let $\mathcal{L}^{r}$ be the collection of $C^{r}, r \geq 0$, Lorenz maps. We endow $\mathcal{L}^{r}$ with a topology that takes care of the domain (P,Q are close), of the exponents and of the coefficients: the coefficients, after a linear rescaling, to make the domains equal, are $C^{r}$ close to each other.

Definition 1.5. Let $\Lambda \subset \mathbb{R}^{2}$ be closed. A Lorenz family is a continuous map $F: \Lambda \rightarrow \mathcal{L}^{r}$, $r \geq 0$

$$
F_{\lambda}=\left\{P_{\lambda}, Q_{\lambda}, \phi_{\lambda}, \psi_{\lambda}\right\}
$$

A Lorenz family $\lambda \in \Lambda \mapsto F_{\lambda}$ is full if given any non-trivial Lorenz map $f \in \mathcal{L}^{2}$ there exists a parameter value $\lambda$ such that $f$ is essentially conjugated to $F_{\lambda}$.

A Monotone Lorenz family is a $C^{3}$ Lorenz family such that

1) $F_{\lambda}$ has negative Schwarzian derivative for all $\lambda \in \Lambda$.

2) $\Lambda=[0,1] \times[0,1]$.

3) $F:(s, t) \rightarrow\left\{-1,1, \phi_{s}, \psi_{t}\right\}$.

4) if $s_{1}<s_{2}$ then $\phi_{s_{1}}(x)<\phi_{s_{2}}(x)$ for all $x \in[-1,0]$ and if $t_{1}<t_{2}$ then $\psi_{t_{1}}(x)<\psi_{t_{2}}(x)$ for all $x \in[0,1]$

5) $\phi_{0}(0)=0, \phi_{1}(0)=1, \psi_{0}(0)=-1$ and $\psi_{1}(0)=0$.

6) $D F_{\lambda}( \pm 1)>1$ for $\lambda \in \Lambda$.

Theorem 1.6. A monotone Lorenz family is a full family.

Next we discuss the notion of renormalization for Lorenz maps. This will allow us to refine the above Theorem.

Definition 1.7. A Lorenz map $f$ is called renormalizable if there exist $P<p<0<q<Q$ such that the first return map to $(p, q)$ is a Lorenz map, say $\left(p, q, f^{a}, f^{b}\right)$. This induced Lorenz map is called a renormalization of $f$. The interval $[p, q]$ is called a domain of renormalization, $(a, b)$ are the periods of renormalization and the type of the renormalization is the pair $(\alpha, \beta)$, where $\alpha$ (resp. $\beta$ ) is the word associated to the branch of $f^{a}$ (resp. $f^{b}$ ) that contains $[p, 0]$ (resp. $[0, q])$.

Let $\mathcal{D}_{\alpha, \beta} \subset \mathcal{L}^{r}$ be the subset of Lorenz maps which have a renormalization of type $(\alpha, \beta)$. These sets are called Domains of Renormalization. In section 3 it will be shown that

1) $\mathcal{D}_{\alpha, \beta}$ is closed and connected,

2) The collection of sets $\mathcal{D}_{\alpha, \beta}$ is nested. If $\mathcal{D}_{\alpha, \beta} \cap \mathcal{D}_{\hat{\alpha}, \hat{\beta}} \neq \emptyset$ then

$$
\mathcal{D}_{\alpha, \beta} \subset \mathcal{D}_{\hat{\alpha}, \hat{\beta}} \text { or } \mathcal{D}_{\hat{\alpha}, \hat{\beta}} \subset \mathcal{D}_{\alpha, \beta} \text {. }
$$


Let $\mathcal{D} \subset \mathcal{L}^{r}$ be the set of renormalizable maps. This set is the union of the nested collection consisting of the sets $\mathcal{D}_{\alpha, \beta}$.

Fix a Lorenz family $F: \Lambda \rightarrow \mathcal{L}^{r}$. This family will intersect the domains $\mathcal{D}_{\alpha, \beta}$, giving rise to the following

Definition 1.8. The archipelago of type $(\alpha, \beta)$ is the set of are all parameter values $A_{\alpha, \beta}$ for which the corresponding Lorenz map is in $\mathcal{D}_{\alpha, \beta}: A_{\alpha, \beta}=F^{-1}\left(\mathcal{D}_{\alpha, \beta}\right)$.

An island in the archipelago $A_{\alpha, \beta}$ is a connected component of the interior of $A_{\alpha, \beta}$.

To express the type of renormalization we are considering, we will speak about $(\alpha, \beta)$ archipelagoes and there $(\alpha, \beta)$-islands.

The archipelagoes inherit properties from the sets $D_{\alpha, \beta}$, they are closed and nested. This implies that the closure of an island defines a Lorenz family, namely the family of the corresponding $(\alpha, \beta)$-renormalizations. We call an island a full island if the induced family is a full Lorenz family.

Theorem 1.9. Every archipelago of a monotone Lorenz family contains a full island.

Observe that Theorem 1.6 is a special case of Theorem 1.9.

Definition 1.10. A Lorenz map is called hyperbolic iff both critical orbits tend to hyperbolic periodic attractors and the complement of the basin of these periodic attractors is a hyperbolic set.

Proposition 1.11. The hyperbolic elements in a monotone Lorenz family form an open and dense set in parameter space.

\section{Conjectures and Remarks}

We finish this section with some conjectures, problems and remarks.

Conjecture 1.12. If $f$ is a $C^{2}$ Lorenz map with exponent $\rho>1$ then the number of periodic homtervals of $f$ is finite.

Definition 1.13. We say that a Lorenz map $f$ has a Cherry attractor if there exists a renormalization of $f,\left(p, q, f^{a}, f^{b}\right)$ with the following properties:

1 . The interval $\left[f^{b}(0+), f^{a}(0-)\right]=\left[p^{\prime}, q^{\prime}\right]$ is invariant under the renormalized map whose restriction $g$ to this interval is one to one but not onto.

2. $g$ has no periodic point.

The $f$-invariant set $\cup_{i=0}^{a} f^{i}\left(\left[p^{\prime}, 0\right] \cup_{j=0}^{q} f^{j}\left(\left[0, q^{\prime}\right]\right.\right.$ is called a Cherry attractor for $f$.

If $J$ is the interval $\left[p^{\prime}, q^{\prime}\right] \backslash g\left(\left[p^{\prime}, q^{\prime}\right]\right.$ then the inverse of $g$ can be extended continuously to the whole interval and gives a map that is constant on $J$, strictly monotone otherwise and maps $p^{\prime}, q^{\prime}$ into 0 . Hence $g^{-1}$ can be thought as a circle map with a flat top without periodic point. This is called a Cherry map and appears as a first return map of a recurrent flow on the torus (see $[\mathrm{MMMS}]$ ). It follows that $J$ is a wandering homterval of $f$. 
Conjecture 1.14. Let $f$ be a $C^{2}$ Lorenz map with exponent $\rho>1$. If $f$ has a wandering homterval then $f$ has a Cherry attractor.

In [MMS] it is proved that smooth interval maps have only finitely many periodic homtervals. Exactly the same proof can be applied to Lorenz maps which do not have wandering intervals. It follows that Conjecture 1.12 is consequence of Conjecture 1.14.

Conjecture 1.15. If $f$ is a $C^{2}$ Lorenz map with exponent $\rho<1$ then:

1) $f$ has at most a finite number of renormalizations.

2) If $f$ is not renormalizable then either there exists a maximal semi conjugacy from $f$ to a piece wise affine Lorenz map with constant derivative ( $\beta$-transformation) or the restriction of $f$ to the interval $[f(0+), f(0-)]$ is $1-1$.

Let $\mathcal{D}$ denotes the set of Lorenz maps that are renormalizable. We prove in section 3 that each connected component of $\mathcal{D}$ is equal to some $\mathcal{D}_{\alpha, \beta}$. From this we can define the renormalization operator $\mathcal{R}: \mathcal{D} \rightarrow \mathcal{L}^{r}$ as follows. Let $f \in \mathcal{D}_{\alpha, \beta}$ and define $\mathcal{R}(f)=A \circ \hat{f} \circ A^{-1}$ where $A(x)=\frac{x}{q}$ and $\hat{f}=\left(p, q, f^{a}, f^{b}\right)$ the $\alpha, \beta$-renormalization of $f$. Hence $\mathcal{R}(f)$ is a Lorenz map with the positive fixed point equal to 1 . If we restrict $\mathcal{R}$ to the space of Lorenz maps with the same normalization we get an operator.

Conjecture 1.16. Let $\mathcal{D}_{i}, i \in \mathbf{N}$ be the connected components of the domain of the renormalization operator.

1) Given a finite sequence $i_{0}, \ldots, i_{n-1}$ of integers not necessarily distinct, there exists a unique normalized Lorenz map g such that $\mathcal{R}^{n}(g)=g$ and $\mathcal{R}^{k}(g) \in \mathcal{D}_{i_{j}}$ whenever $k=i_{j}$ $\bmod n$, compare $[A C T]$.

2) If $f$ is a normalized Lorenz map such that $\mathcal{R}^{k}(f) \in \mathcal{D}_{i_{j}}$ whenever $k=i_{j} \bmod n$ then $\mathcal{R}^{k n}(f)$ converges exponentially fast to $g$ as $k \rightarrow \infty$, compare [ACT].

3) Let $f$ be a Lorenz map whose forward orbit under the renormalization operator meets only a finite number of connected components of the domain. Then there exists a compact set $\mathcal{K} \subset \mathcal{L}$ such that $\mathcal{R}^{n}(f)$ belongs to $\mathcal{K}$ for all $n$. In particular, the length of the left component of the domain of $\mathcal{R}^{n}(f)$ is bounded from above and from below.

4) Let $\mathcal{B} \subset \mathcal{D}$ be the union of a finite number of connected components of $\mathcal{D}$. Then there exists a compact set $\mathcal{K}_{\mathcal{B}}$ of the space of Lorenz maps such that for any mapping $f \in \mathcal{B}$ whose forward orbit remains in $\mathcal{B}$, there exists $n_{0}$ such that for $n \geq n_{0}$, $\mathcal{R}^{n}(f) \in \mathcal{K}_{\mathcal{B}}$.

Conjecture 1.17. There exist a monotone Lorenz family such that each archipelago is an island.

Conjecture 1.18. For generic Lorenz families the following holds: there exists a bound, depending on the family, for the number of island in each archipelago.

In $[\mathrm{MP}]$ it has been proved that in a $C^{1}$ generic one-parameter family of $C^{2}$ circle difeomorphisms the rotation number is a piecewise monotone function. Conjecture 1.18 is the 
corresponding statement for Lorenz families.

Conjecture 1.19. For a monotone Lorenz family the diameter of the islands goes to zero as the period of renormalization goes to infinity.

Conjecture 1.20. For a monotone Lorenz family, the set of parameter values that belong to infinitely many archipelagoes has Lebesgue measure zero.

We say that an island is of generation 0 if it is not contained in another island. By induction we say that an island is of generation $n$ if it is not of generation $n-1$ and any island that contains it is of generation $\leq n-1$. From theorem 1.11 it follows that any full island of generation $n$ contains infinitely many island of generation $n+1$. In particular there are uncountably many parameter values that corresponds to infinitely renormalizable islands. This is in sharp contrast with Conjecture 1.15 .

From $[\mathrm{R}]$ it follows that for generic two parameter families of Lorenz maps, the set of parameter values corresponding to maps that have positive Lyapunov exponents has positive Lebesgue measure.

Conjecture 1.21. For generic two parameter families of Lorenz maps the set of parameter values corresponding to maps that are not hyperbolic and that do not have positive Lyapunov exponents has zero Lebesgue measure.

Acknowledgements. Part of this work was written while the first author was visiting IMPA and the second author was visiting IHES and the graduate center of CUNY. The authors would like to thank these institutions for their kind hospitality.

\section{Notation}

Let $I \subset[-1,1]$ be an interval with boundary points $a$ and $b$, say $a<b$. Then $\partial I=\{a, b\}$, $\partial_{-} I=\{a\}$ and $\partial_{+} I=\{b\}$. If $J \subset I \subset[-1,1]$ are two intervals and $\partial_{-} J=\partial_{-} I$, we say $J \subset_{l} I$. If $\partial_{+} J=\partial_{+} I$ then we say $J \subset_{r} I$. In the case that $\partial J \cap \partial I=\emptyset$ we write $J \subset_{\text {int }} I$. The discontinuity of Lorenz maps in 0 causes that such maps has two critical orbits:

$$
f^{n}\left(0_{-}\right)=\lim _{x \uparrow 0} f^{n}(x) \text { and } f^{n}\left(0_{+}\right)=\lim _{x \downarrow 0} f^{n}(x),
$$

with $n \geq 0$.

\section{Combinatorial Properties of Lorenz-maps}

We will start by defining kneading sequences for Lorenz maps, similarly as was done in $[\mathrm{MT}]$ for continuous piecewise monotone interval maps. Clearly, to describe Lorenz maps 
we will need two kneading sequences, one for $0_{-}$and one for $0_{+}$. Fix a Lorenz map $f:[-1,1] \rightarrow[-1,1]$

Let $\mathbb{B}_{n}(f)$ be the collection of branches of $f^{n}$. That is, the collection of maximal intervals on which $f^{n}$ is monotone. If $I \in \mathbb{B}_{n}(f)$ then the word $\omega(I) \in\{L, R\}^{n}$ is such that

$$
\begin{aligned}
& \omega_{i}(I)=L \text { if } f^{i}(I) \subset[-1,0) \\
& \omega_{i}(I)=R \text { if } f^{i}(I) \subset(0,1]
\end{aligned}
$$

for $0 \leq i<n$. The kneading sequences are defined as

$$
K_{n}^{-}(f)=\omega\left(I_{-}(n)\right) \text { and } K_{n}^{+}(f)=\omega\left(I_{+}(n)\right)
$$

where $I_{-}(n), I_{+}(n) \in \mathbb{B}_{n}(f), n \geq 0$, with $\partial_{+} I_{-}(n)=0$ and $\partial_{-} I_{+}(n)=0$. Let $K^{-}(f)$ and $K^{+}(f)$ be the limits of respectively $K_{n}^{-}(f)$ and $K_{n}^{+}(f), n \rightarrow \infty$.

For every branch $I \in \mathbb{B}_{n}(f)$ there exist unique cutting times $l_{n}(I, f)$ and $r_{n}(I, f)$ such that

$$
0 \in \partial_{+} f^{l_{n}(I, f)}(I) \text { and } 0 \in \partial_{-} f^{r_{n}(I, f)}(I) .
$$

When it is clear which map is under consideration, we will suppress the symbol $f$ in the above notation.

Lemma 2.1. Let $f$ and $g$ be Lorenz maps such that $K_{n}^{ \pm}(f)=K_{n}^{ \pm}(g)$ for some $n \geq 1$ and assume that

$$
f^{i}\left(0_{ \pm}\right)=0 \Leftrightarrow g^{i}\left(0_{ \pm}\right)=0
$$

where $i \leq n$.

There exists an orientation preserving homeomorphism $h:[-1,1] \rightarrow[-1,1]$ such that

1) $h$ preserves branches and their type: for every $k \leq n, I \in \mathbb{B}_{k}(f)$

$$
h(I) \in \mathbb{B}_{k}(g) \text { and } \omega(I, f)=\omega(h(I), g) .
$$

2) if $I \in \mathbb{B}_{k}(f)$ and $f(I)=I^{\prime} \in \mathbb{B}_{k-1}(f)$ then

$$
g(h(I))=h\left(I^{\prime}\right) \in \mathbb{B}_{k-1}(g) .
$$

3) if $I \in \mathbb{B}_{k}(f)$ and $f(I) \subset_{l / r} I^{\prime} \in \mathbb{B}_{k-1}(f)$ then

$$
g(h(I)) \subset_{l / r} h\left(I^{\prime}\right) \in \mathbb{B}_{k-1}(g) .
$$

4) for $k \leq n, I \in \mathbb{B}_{k}(f)$

$$
l_{k}(I, f)=l_{k}(h(I), g) \text { and } r_{k}(I, f)=r_{k}(h(I), g) .
$$

Proof. First we will show that 2), 3) and 4) follow from 1).

$1) \Rightarrow 2), 3)$. Let $I=(x, y) \in \mathbb{B}_{k}(f)$ and assume $f(I) \subset I^{\prime}=\left(x^{\prime}, y^{\prime}\right) \in \mathbb{B}_{k-1}(f)$. It suffices to prove 


\section{Claim.}

$$
\begin{aligned}
& f(x)=x^{\prime} \Leftrightarrow g(h(x))=h\left(x^{\prime}\right) \\
& f(y)=y^{\prime} \Leftrightarrow g(h(y))=h\left(y^{\prime}\right)
\end{aligned}
$$

By symmetry we only have to consider the left boundary. First observe that $f(x)=x^{\prime}$ whenever $x \neq 0$. So if $x \neq 0$ then $h(x) \neq 0$ and $g(h(x))=h\left(x^{\prime}\right)$. The Claim is proved in this case.

Assume $x=0$ and $f(x)=x^{\prime}$. Because $x^{\prime}$ is a boundary point of $I^{\prime} \in \mathbb{B}_{k-1}(f)$ there exists $j \leq k-1$ such that $f^{j}\left(0_{+}\right)=0$. By assumption, the same holds for $g: g^{j}\left(0_{+}\right)=0$. Now assume by contradiction that $g(h(x)) \neq h\left(x^{\prime}\right)$. Then $g\left(0_{+}\right)=g(h(x))$ belongs to the interior of $g\left(h\left(I^{\prime}\right)\right)$ and $g^{j-1}\left(h\left(I^{\prime}\right)\right)$ contains 0 in its interior. Therefore, $g^{j} \mid h\left(I^{\prime}\right)$ is not monotone. This is a contradiction because $h\left(I^{\prime}\right) \in \mathbb{B}_{k-1}(g)$ and $j \leq k-1$. We proved that $g(h(x))=h\left(x^{\prime}\right)$.

$(1) \Rightarrow 2), 3))$

$1) \Rightarrow 4)$. Observe that for each $I \in \mathbb{B}_{k}(f)$ and $i \leq k$ there exists $I^{\prime} \in \mathbb{B}_{k-i}(f)$ such that one of the following three possibilities holds:

a) $f^{i}(I)=I^{\prime}$,

b) $f^{i}(I) \subset_{l} I^{\prime}$ or $f^{i}(I) \subset_{r} I^{\prime}$,

c) $f^{i}(I) \subset_{\text {int }} I^{\prime}$.

Moreover observe that there are unique numbers $0 \leq i_{1}<i_{2}<k$ such that for $0 \leq i<i_{1}$ $f^{i}(I)$ is in case a). For $i_{1} \leq i<i_{2} f^{i}(I)$ is in case b), say $f^{i}(I) \subset_{l} I^{\prime}$. Finally for $i \geq i_{2}$ $f^{i}(I)$ is in case $\left.\mathrm{c}\right)$. Clearly this numbers are exactly the cutting times

$$
\begin{aligned}
& i_{1}=r_{k}(I, f) \text { and } i_{2}=l_{k}(I, f) \text { or } \\
& i_{1}=l_{k}(I, f) \text { and } i_{2}=r_{k}(I, f) .
\end{aligned}
$$

Using properties 2) and 3) of the Lemma it follows that the intervals $g^{i}(h(I))$ jump exactly at the same times $i_{1}$ and $i_{2}$ from case to case. Hence

$$
\begin{aligned}
& r_{k}(h(I), g)=i_{1}=r_{k}(I, f) \text { and } l_{k}(h(I), g)=i_{2}=l_{k}(I, f) \text { or } \\
& r_{k}(h(I), g)=i_{2}=r_{k}(I, f) \text { and } l_{k}(h(I), g)=i_{1}=l_{k}(I, f) .
\end{aligned}
$$

$$
(1) \Rightarrow 4))
$$

The proof of property 1 ) will be by induction in $n \geq 1$. It clearly holds for $n=1$. Assume 1 ) holds for some $n \geq 1$. In particular we may assume that also 2), 3) and 4) hold for this $n \geq 1$.

Observe that the collection of branches $\mathbb{B}_{k}(f)$ with $k=0,1,2, \ldots, n$ define a refining sequence of partitions of $[-1,1]$. The homeomorphism $h$ maps this sequence of partitions to the partitions formed by $\mathbb{B}_{k}(g)$ with $k \leq n$. To prove 1$)$ for $n+1$ we have to construct a homeomorphism $H$ which also preserves the above partitions and moreover maps $\mathbb{B}_{n+1}(f)$ 
to $\mathbb{B}_{n+1}(g)$. In particular $H$ is obtained by redefining $h$ in the interior of the branches $\mathbb{B}_{n}(f)$.

Consider a branch $I \in \mathbb{B}_{n}(f)$ with $h(I)=I^{\prime} \in \mathbb{B}_{n}(g)$. The new homeomorphism $H$ will also satisfy $H(I)=I^{\prime}$. The boundary of the interval $f^{n}(I)$ consists of the critical values

$$
\partial f^{n}(I)=\left\{f^{n-l_{n}(I, f)}\left(0_{+}\right), f^{n-r_{n}(I, f)}\left(0_{-}\right)\right\} .
$$

Property 4) of the Lemma states that also

$$
\partial g^{n}\left(I^{\prime}\right)=\left\{g^{n-l_{n}(I, f)}\left(0_{+}\right), g^{n-r_{n}(I, f)}\left(0_{-}\right)\right\},
$$

the cutting times of $I$ and $I^{\prime}$ are the same.

Now we will use that the kneading sequences of $f$ and $g$ are the same up to $n+1$. Let $\left(\theta_{i}\right)_{i=1, \ldots, n+1}=K_{n}^{ \pm}(f)=K_{n}^{ \pm}(g)$. Observe that

$$
I \notin \mathbb{B}_{n+1}(f) \Longleftrightarrow \theta_{n-r_{n}(I, f)}=R \text { and } \theta_{n-l_{n}(I, f)}=L .
$$

And

$$
I^{\prime} \notin \mathbb{B}_{n+1}(g) \Longleftrightarrow \theta_{n-r_{n}(I, g)}=R \text { and } \theta_{n-l_{n}(I, g)}=L .
$$

In particular we have

$$
I \in \mathbb{B}_{n+1}(f) \Longleftrightarrow I^{\prime} \in \mathbb{B}_{n+1}(g) .
$$

If $I \in \mathbb{B}_{n+1}(f)$ we do not have to change $h: H \mid I=h$. If $I \notin \mathbb{B}_{n+1}(f)$ then both $I$ and $I^{\prime}$ will have two branches of respectively $\mathbb{B}_{n+1}(f)$ and $\mathbb{B}_{n+1}(g)$. Define $H \mid I$ such that these two sub-branches are matched.

Once this construction has been done for all branches $I \in \mathbb{B}_{n}(f)$ we will obtain a homeomorphism $H$ which preserves the branches in $\mathbb{B}_{n+1}(f)$ and their types.

(Lemma 2.1)

Example. Let $\phi_{\lambda}:[-1,1] \rightarrow[-1,1], \lambda \in[0,1]$, be a one parameter family of unimodal maps. Say, $\phi_{\lambda}( \pm 1)=-1$ for $\lambda \in[0,1]$, and $\phi_{0}(0)=0, \phi_{1}(0)=1$. Moreover, assume that this unimodal family is monotone: whenever $\lambda_{1}<\lambda_{2}$ we have $\phi_{\lambda_{1}}(t)<\phi_{\lambda_{2}}(t)$ for all $t \in(-1,1)$.

Consider the Lorenz family $f_{x, y}:[-1,1] \rightarrow[-1,1]$ with $(x, y) \in[0,1] \times[0,1]$ and

$$
\begin{aligned}
& f_{(x, y)}(t)=\phi_{x}(t) \text { if } t<0 \\
& f_{(x, y)}(t)=\phi_{1-y}(t) \text { if } t>0 .
\end{aligned}
$$

Notice that for $(x, y) \in U=\{(t, s) \in[0,1] \times[0,1] \mid s=1-t\}$ the Lorenz map $f_{(x, y)}$ behaves exactly like the Unimodal map $\phi_{x}$. Namely

$$
f_{(x, y)}^{n}(t)= \pm \phi_{x}^{n}(t)
$$


for $n \geq 0$ and $t \in[-1,1]$.

This example inspires to change the coordinates of the parameter space of monotone Lorenz families. Fix a monotone Lorenz family $F:[0,1] \times[0,1] \rightarrow \mathcal{L}$. The usual coordinates $x$ and $y$ for $[0,1] \times[0,1]$ are not appropriate to explore the monotonicity of the family. We will use

$$
\begin{aligned}
U & =\{(x, y) \mid y=1-x\} \\
M & =\{(x, y) \mid y=x\} .
\end{aligned}
$$

as coordinate axis for $[0,1] \times[0,1]$ giving rise to the coordinates $u$ and $m$ on $[0,1] \times[0,1]$, $u=x-y, m=x+y-1$.

For $u \in U$ let $M_{u}=\{(u, m) \mid m \in M\}=\{(x, y) \mid x-y=u\}$ and for $(x, y) \in[0,1] \times[0,1]$ let

$$
\begin{aligned}
& C_{(x, y)}^{+}=\{(t, s) \mid t \geq x, s \geq y\}, \\
& C_{(x, y)}^{-}=\{(t, s) \mid t \leq x, s \leq y\}, \\
& B_{(x, y)}=[0,1] \times[0,1] \backslash\left(C_{(x, y)}^{+} \cup C_{(x, y)}^{-}\right) .
\end{aligned}
$$
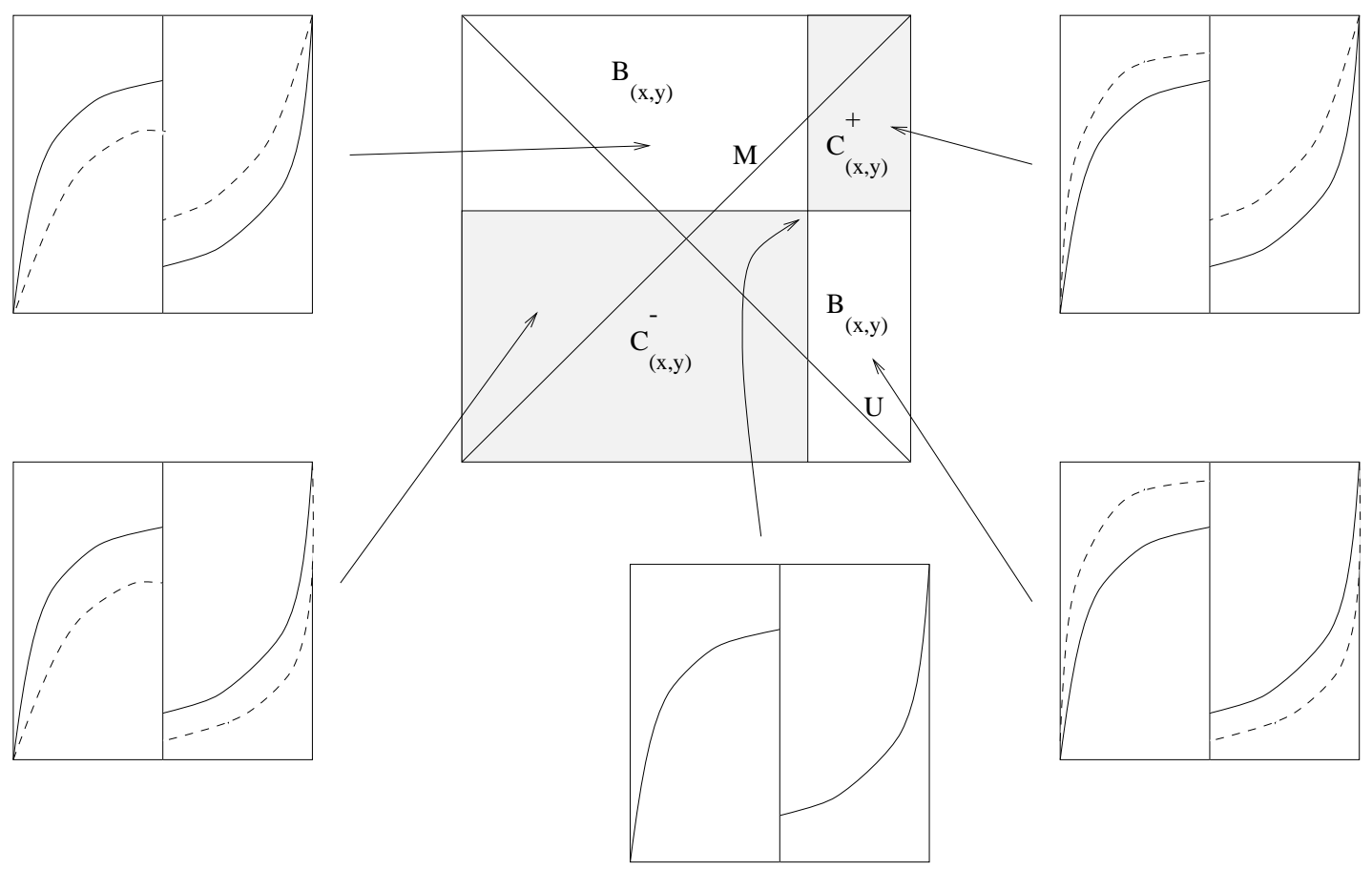

Figure 1 The $u, m$-coordinates

These cones will play a crucial role in the study of the parameter space of monotone Lorenz families. The $C^{+}$-cones describe deformations in which both branches move up. The $C^{-}$-cones describe deformations in which both branches move down. The $B$-cone contains the maps for which one branch moves up and the other down. The situation is 
illustrated in Figure 1. The deformations into the $B$-cones are the ones which are difficults to understand. We will explore the deformations into the $C^{ \pm}$-cones.

For example, the monotonicity of the family implies immediately the monotonicity in kneading information: let $K^{ \pm}(z)=K_{F(z)}^{ \pm}$then

$$
K^{ \pm}\left(z^{\prime}\right) \geq K^{ \pm}(z) \text { for } z^{\prime} \in C^{+}(z)
$$

and

$$
K^{ \pm}\left(z^{\prime}\right) \leq K^{ \pm}(z) \text { for } z^{\prime} \in C^{-}(z)
$$

where we used the usual lexicographic order on the $L-R$-sequences $(L<R)$.

Proof of Proposition 1.11. Given a map $f$ in a monotone Lorenz family we will construct arbitrarily close a map in the family whose critical orbits tend to hyperbolic periodic attractors. Then general arguments $([\mathrm{M}])$ will show that this perturbation is actually a hyperbolic Lorenz map.

Assume that both critical orbits do not tend to a periodic attractor. We also may assume that the map $f$ is not in the boundary of parameter space: $C_{f}^{-} \cap([0,1] \times[0,1]) \neq \emptyset$. The first step will be to find a map in $C_{f}^{-}$close to $f$ such that $0_{-}$is periodic.

Take $g_{1} \in C_{f}^{-}$close to $f$. Assume that there is some minimal $n_{0}>0$ such that $g_{1}^{n_{0}}(0)<$ $0<f^{n_{0}}\left(0_{-}\right)$. If we move back along the straight line from $g_{1}$ to $f$ then $g^{n_{0}}\left(0_{-}\right)$will increase up to $f^{n_{0}}\left(0_{-}\right)$. Hence along this straight line there is a map $g$ with $g^{n_{0}}\left(0_{-}\right)=0$. The second case is that for all $n>0$ we have $g_{1}^{n}\left(0_{-}\right)$and $f^{n}\left(0_{-}\right)$are on the same side of 0 . In particular $g_{1}^{n}\left(0_{-}\right)<f^{n}\left(0_{-}\right)$for all $n>0$. Consider the intervals

$$
J_{n}=\left[g_{1}^{n}\left(0_{-}\right), f^{n}\left(0_{-}\right)\right]
$$

with $n \geq 0$. Observe $f\left(J_{n}\right) \subset J_{n+1}$. This interval $J_{n}$ has to accumulate at $0_{+}$. If not the interval $J_{1}$ would be a hometerval of $f$ not accumulating on $0_{+}$. Then we can define a continuous $C^{2}$ map, by redefining $f$ on a small interval $(0, a]$, having $J_{1}$ as a hometerval: by [MMS] we know that $J_{1}$ tends to a periodic attractor of $f$. In particular the orbit $f^{n}\left(0_{-}\right)$tends to a periodic attractor. This is a contradiction because we assumed both critical orbits not to accumulate at periodic attractors.

We proved that the critical orbit $g_{1}^{n}\left(0_{-}\right)$accumulates at $0_{+}$. For each perturbation $g_{2} \in C_{g_{1}}^{-}$ there will be some $n_{0}>0$ such that $g_{2}^{n_{0}}\left(0_{-}\right)<0<g_{1}^{n_{0}}\left(0_{-}\right)$. Again we can move back a little bit to find a map $g \in C_{f}^{-}$close to $f$ such that $0_{-}$is periodic for $g$. We finished the proof of

Claim. If both critical orbits of $f$ are not accumulating at a periodic attractor then arbitrarily close there is a map $g$ such that $0_{-}$is periodic. In particular arbitrarily close 
there is a map such that $0_{-}$is attracted to a hyperbolic periodic attractor but is not itself a periodic attractor.

Assume that one critical orbit is attracted to a periodic attractor but is not itself periodic. We may assume that this orbit is the of the critical point $0_{-}$. The boundary point -1 and 1 are expanding fixed points. Because the map has negative Schwarzian derivative the periodic attractor is of one of the following types.

1) There exist an interval $(0, p]$ and $n>0$ such that $f^{n}((0, p)) \subset(0, p)$ and $f^{n}(p)=p$. By taking $g \in C_{f}^{-}$close to $f$ we may assume that the periodic attractor is hyperbolic and still attracts also $0_{-}$. We got a situation in which both critical orbits are attracted to a hyperbolic periodic attractor. The Proposition 1.11 is proved.

2) There exist an interval $[p, 0)$ and $n>0$ such that $f^{n}([p, 0)) \subset[p, 0)$. Again by taking $g \in C_{f}^{+}$we may assume that the periodic orbit of $p$ is a hyperbolic attractor. This situation will persist in a small neighborhood of $g$.

Left is to deform $g$ such that also $0_{+}$is attracted towards a hyperbolic attractor. Assume that $0_{+}$is not attracted towards a hyperbolic attractor. It could be attracted towards a neutral periodic attractor. Take $g_{1} \in C_{g}^{+}$close to $g$. Consider the two orbits $g^{n}\left(0_{+}\right)$and $g_{1}^{n}\left(0_{+}\right), n \geq 1$. If at some moment these points are separated then we can move back a little bit towards $g$ and make $0_{+}$periodic and by moving back a little bit more we can make it to be attracted towards the periodic attractor which already attracted $0_{-}$.

If these points are never separated we show, as before, that they accumulate at $0_{+}$or is attracted towards a periodic attractor. As before we can make $0_{+}$to be attracted towards a hyperbolic attractor.

(Proposition 1.11)

The proof of Proposition 1.11 also shows that the hyperbolic Lorenz map are dense in the whole space $\mathcal{L}^{r}$ of Lorenz maps (this can also be obtained from the arguments of $[\mathrm{R}]$ ). Notice that this space contains a closed subspace $\mathcal{L}_{s}$ of symmetric Lorenz maps. These are Lorenz maps $f$ with branches $f_{-}$and $f_{+}$such that $f_{-}(x)=-f_{+}(-x)$. To each symmetric Lorenz map we can associate a unimodal map $\phi_{f}$, namely

$$
\begin{aligned}
& \phi_{f}(x)=f_{-}(x) \text { for } x<0 \\
& \phi_{f}(x)=-f_{+}(x) \text { for } x \geq 0 .
\end{aligned}
$$

Observe that $\phi^{n}(x)= \pm f^{n}(x)$, for $n \geq 0$. The Lorenz map $f$ can be seen as an orientation preserving "lift" of the unimodal map $\phi_{f}$. It follows that the density of the hyperbolic Lorenz maps in $\mathcal{L}_{s}$ would imply the density of hyperbolic maps in the space of unimodal maps. This is an important open question.

One should compare this with the situation of flows on non-orientable surfaces. These flows are covered by flows on the orientable cover of the manifold. Proposition 1.11 should be compared with the Peixoto Theorem for flows on orientable surfaces. 


\section{Domains of Renormalization}

In this section we will study some topological properties of the sets $\mathcal{D}_{\alpha, \beta}$, the Lorenz maps which have a $(\alpha, \beta)$-renormalization.

Lemma 3.1. Let $p<0<q$ be two periodic points of the Lorenz map $f$. Say with period $a$ and $b$ respectively. Assume

1) $(p, q) \cap(\operatorname{orb}(p) \cup \operatorname{orb}(q))=\emptyset$.

2) $f^{a} \mid[p, 0]$ and $f^{b} \mid[0, q]$ are monotone.

3) $f^{a}([p, 0]) \supset[p, 0]$ and $f^{b}([0, q]) \supset[0, q]$.

Let $(l, 0)$ (resp. $(0, r))$ be the maximal interval on which $f^{a}$ (resp $\left.f^{b}\right)$ is monotone.

Then

$$
f^{a}(l) \leq l \text { and } f^{b}(r) \geq r .
$$

Furthermore if $f^{a}(l)=l$ (resp. $\left.f^{b}(r)=r\right)$ then $f^{b}\left(0_{+}\right)=0\left(\right.$ resp. $\left.f^{a}\left(0_{-}\right)=0\right)$.

In particular, if the Lorenz map has negative Schwarzian derivative then the periodic points $p$ and $q$ are hyperbolic repellors.

proof. Assume that $f^{a}(l) \geq l$. Let $L=(l, p)$. The assumption implies that $f^{a}(L) \subset L$. Consequently we get that $\left.f^{n}\right|_{L}$ is monotone for all $n \geq 0, L$ is a homterval.

The maximality of the interval $(l, 0)$ gives some $i<a$ such that $0 \in \partial f^{i}(L)$. From property 1 , it follows that $f^{i}(L) \supset(0, q)$. In particular $(0, q)$ is a homterval. This is only possible if $f^{b}\left(0_{+}\right)=0$, the point $0_{+}$is periodic. Hence $f^{a}(L)=L$ and therefore, $f^{a}(l)=l$.

Let $L_{-}=L=(l, p)$ and $L_{+}=(p, 0)$. We have $f^{a}\left(L_{ \pm}\right) \supset L_{ \pm}$. The Maximal Principle for maps with negative Schwarzian derivative, see $[\mathrm{MS}]$, implies that $p$ is a repelor.

(Lemma 3.1)

Lemma 3.2. If $f \in \mathcal{D}_{\alpha, \beta} \cap \mathcal{D}_{\hat{\alpha}, \hat{\beta}}$ then $\hat{\alpha}$ and $\hat{\beta}$ are formed by concatenating the words $\alpha, \beta$. In particular

$$
\begin{aligned}
& \hat{\alpha}=\alpha \beta \ldots \\
& \hat{\beta}=\beta \alpha \ldots
\end{aligned}
$$

and $|\hat{\alpha}|,|\hat{\beta}| \geq|\alpha|+|\beta|$.

Or vice versa, $\alpha$ and $\beta$ can be expressed in terms of $\hat{\alpha}$ and $\hat{\beta}$.

Proof. Let $f \in \mathcal{D}_{\alpha, \beta} \cap \mathcal{D}_{\hat{\alpha}, \hat{\beta}}$ with renormalizations $\left(p, q, f^{a}, f^{b}\right)$ and $\left(\hat{p}, \hat{q}, f^{\hat{a}}, f^{\hat{b}}\right)$ of type respectively $(\alpha, \beta)$ and $(\hat{\alpha}, \hat{\beta})$. We will first show that

$$
(\hat{p}, \hat{q}) \subset(p, q)(\text { or }(p, q) \subset(\hat{p}, \hat{q}))
$$

Without loss of generality we may assume that $(p, q)$ is not contained in $(\hat{p}, \hat{q})$, say $\hat{p} \in(p, 0)$. We are going to show that $\hat{q} \in(0, q)$, the intervals are nested. 
Assume $\hat{q}>q$. Then

$$
f^{\hat{b}}(q) \in f^{\hat{b}}((0, \hat{q})) \subset(\hat{p}, \hat{q}) \subset(p, \hat{q}) .
$$

However, the orbit of $q$ never enters $(p, q)$. So $f^{\hat{b}}(q) \in[q, \hat{q})$. Because the orbit of $q$ is finite it is impossible that $f^{\hat{b}}(q) \in(q, \hat{q})$ : the map $f^{\hat{b}}:[q, \hat{q}] \rightarrow[q, \hat{q}]$ is monotone and would have a periodic attractor. So $f^{\hat{b}}(q)=q$ and $\hat{b}=m \cdot b$. The interval $[q, \hat{q}]$ is periodic which is impossible because the orbits in the boundary are expanding, see Lemma 3.1. We proved $\hat{q} \leq q$.

The aim is to prove that $\hat{q}<q$. Assume $\hat{q}=q$. Then both renormalizations will have $\left.f^{b}\right|_{(0, q)}$ as right branch. In particular $f^{b}\left(0_{+}\right)>\hat{p}$.

Let $(x, 0) \subset(p, 0)$ be the maximal monotone interval on which $f^{\hat{a}}$ is monotone. The map $f$ admits a renormalization of type $(\hat{\alpha}, \hat{\beta})=(\hat{\alpha}, \beta)$ we have

1) $\hat{p} \in(x, 0)$,

2) $f^{\hat{a}}((x, 0)) \supset(x, 0)$ (by Lemma 3.1).

Observe that $f^{a}((x, 0)) \subset(0, q)$. The next images of this interval will always stay on the right side of $f^{b}\left(0_{+}\right)$. We have

$$
f^{b}\left(0_{+}\right)>\hat{p}>x,
$$

the images of $(x, 0)$ will never be able to cover $(x, 0)$ completely, contradiction. We proved $\hat{q}<q$. In particular that the $(\hat{\alpha}, \hat{\beta})$ renormalization is a first return map of the $(\alpha, \beta)$ renormalization.

(Lemma 3.2)

In the proof of the next Proposition we will use the usual lexicographic order on the $0-1-$ words. The length of a word $\omega$ will be denoted by $|\omega|$. Moreover, if $j<\min \left\{\left|\omega_{1}\right|,\left|\omega_{2}\right|\right\}$ then

$$
\omega_{1}<{ }_{j} \omega_{2}
$$

means that $\omega_{1}<\omega_{2}$ and the words differ in the first $j$ symbols.

Proposition 3.3. The domains of renormalization have the following properties

1) $\mathcal{D}_{\alpha, \beta}$ is closed and connected,

2) The collection of sets $\mathcal{D}_{\alpha, \beta}$ is nested. If $\mathcal{D}_{\alpha, \beta} \cap \mathcal{D}_{\hat{\alpha}, \hat{\beta}} \neq \emptyset$ then

$$
\mathcal{D}_{\alpha, \beta} \subset \mathcal{D}_{\hat{\alpha}, \hat{\beta}} \text { or } \mathcal{D}_{\hat{\alpha}, \hat{\beta}} \subset \mathcal{D}_{\alpha, \beta} .
$$

proof. The sets $\mathcal{D}_{\alpha, \beta}$ are closed because of Lemma 3.1. Let $f, g \in \mathcal{D}_{\hat{\alpha}, \hat{\beta}}$ and assume that $f \in \mathcal{D}_{\alpha, \beta}$. To prove the Proposition we have to show that also $g \in \mathcal{D}_{\alpha, \beta}$.. Observe that, by Lemma 3.2, the kneading sequences of $f$ and $g$ are equal up to at least $|\alpha|+|\beta|=a+b$.

Let $\left(p, q, f^{a}, f^{b}\right)$ be the $(\alpha, \beta)$ renormalization of $f$ and $(0, y)$ the maximal interval on which $f^{b}$ is monotone. Lemma 3.1 states that $f^{b}((0, y)) \supset(0, y)$. In particular $f^{b}(y)>y$ 
There exists a unique $j=r_{b}((0, y), f)<b$, which by Lemma 2.1 depends only on $\alpha$ and $\beta$, such that $0=\partial_{+} f^{j}((0, y))$. The kneading sequence of $f^{b}(y)$ equals $\sigma^{b-j}(\hat{\alpha})$ and satisfies

$$
\sigma^{b-j}(\hat{\alpha})>_{j} \beta,
$$

which is the combinatorial formulation of $f^{b}(y)>y$. Observe that

$$
\left|\sigma^{b-j}(\hat{\alpha})\right|=\hat{a}-(b-j) \geq a+b-(b-j)>j,
$$

the above relation between $\sigma^{b-j}(\hat{\alpha})$ and $\beta$ is well defined.

The map $g$ has a $(\hat{\alpha}, \hat{\beta})$ renormalization. Lemma 3.2 states that $\hat{\beta}=\beta \alpha \cdots$. The map $g$ has a branch $\left(0, y^{\prime}\right)$ of $g^{b}$ of type $\beta$. We are going to prove that

$$
g^{b}\left(\left(0, y^{\prime}\right)\right) \supset\left(0, y^{\prime}\right) .
$$

First, because $\hat{\beta}=\beta \alpha$ we have $g^{b}\left(0_{+}\right)<0$. The branch $\left(0, y^{\prime}\right)$ is going to be cut also at moment $j=r_{b}\left(\left(0, y^{\prime}\right), g\right)=r_{b}((0, y), f)$. So the kneading sequence of $g^{b}\left(y^{\prime}\right)$ equals

$$
\sigma^{b-j}(\hat{\alpha})>_{j} \beta .
$$

In particular $g^{b}\left(y^{\prime}\right)>y^{\prime}$, otherwise the kneading sequence $\sigma^{b-j}(\hat{\alpha})$ would start with the word $\beta$. This contradicts the kneading information obtained from $f$. We proved that $g^{b}\left(\left(0, y^{\prime}\right)\right) \supset\left(0, y^{\prime}\right)$ and hence the existence of a periodic point $q^{\prime} \in\left(0, y^{\prime}\right)$ with $g^{b}\left(q^{\prime}\right)=q^{\prime}$. Let $\left(x^{\prime}, 0\right)$ be the maximal interval on which $g^{a}$ is monotone, the branch of type $\alpha$. In a similar way as above we show that $f^{a}\left(\left(x^{\prime}, 0\right)\right) \supset\left(x^{\prime}, 0\right)$ and the existence of a periodic point $p^{\prime} \in\left(x^{\prime}, 0\right)$ with $g^{a}\left(p^{\prime}\right)=p^{\prime}$.

Left is to show that $\left(p^{\prime}, q^{\prime}, g^{a}, g^{b}\right)$ is an $(\alpha, \beta)$ renormalization. First we will show that the orbits of $p^{\prime}$ and $q^{\prime}$ never enters $\left(p^{\prime}, q^{\prime}\right)$. The kneading sequences of the periodic orbits of $p^{\prime}$ and $q^{\prime}$ are respectively $\alpha^{\infty}$ and $\beta^{\infty}$. The periodic orbits of $p$ and $q$ of $f$ do never enter in the interval $(p, q)$ and also have kneading sequences respectively $\alpha^{\infty}$ and $\beta^{\infty}$. This implies the following kneading information: for every $k \geq 0$ it is impossible that

$$
\alpha^{\infty}<\sigma^{k}\left(\beta^{\infty}\right), \sigma^{k}\left(\alpha^{\infty}\right)<\beta^{\infty} .
$$

This kneading information implies that the orbits of $p^{\prime}$ and $q^{\prime}$ never enter $\left(p^{\prime}, q^{\prime}\right)$.

Left is to show that $g^{b}\left(\left(0, q^{\prime}\right)\right) \subset\left[p^{\prime}, q^{\prime}\right]$ (and $\left.g^{a}\left(\left(p^{\prime}, 0\right)\right) \subset\left[p^{\prime}, q^{\prime}\right]\right)$. Because $g$ has an $(\hat{\alpha}, \hat{\beta})$ renormalization there exists a branch $\hat{q} \in(0, z) \subset\left(0, q^{\prime}\right)$ with kneading sequence $\hat{\beta}=\beta \alpha \ldots$ If $g^{b}\left(0_{+}\right)$is left of $p^{\prime}$ then also $g^{b}\left((0, z)\right.$ is left of $p^{\prime}$ ( the orbit of $p^{\prime}$ never enters $\left.\left(p^{\prime}, q^{\prime}\right)\right)$. According to the word $\hat{\beta}=\beta \alpha \alpha \ldots$ we have to apply a few times the branch $g^{a} \mid\left(x^{\prime}, 0\right)$. Then $g^{b+i \cdot a}((0, z))$ will still be on the left side of $p^{\prime}$. This is impossible. Either $b+i \cdot a=\hat{b}$, in which case $g^{b+i \cdot a}(\hat{q})=\hat{q}$ but $g^{b+i \cdot a}(\hat{q})<p^{\prime}$. Or $b+i \cdot a<\hat{b}$, in which case we have to apply the branch $g^{b} \mid(0, y)$ but $g^{b+i \cdot a}((0, z)) \cap(0, y)=\emptyset$. We proved that $\left(p^{\prime}, q^{\prime}, g^{a}, g^{b}\right)$ is a $(\alpha, \beta)$ renormalization.

(Proposition 3.3) 


\section{Realization of Finite Combinatorics}

Proposition 4.1. Let $V=[-1,0] \times[0,1]$ and $\Lambda \subset \mathbf{R}^{2}$ be homeomorphic to $V$. Let

$$
\Lambda \ni \lambda \mapsto f_{\lambda}:[-1,1] \rightarrow[-1,1]
$$

be a family of Lorenz maps satisfying the following properties:

1) the branches $f_{\lambda, \pm}$ are $C^{1}, f_{\lambda}( \pm 1)= \pm 1$, and there exists $K>0$ such that $0<D f_{\lambda}(x) \leq$ $K$ for all $x \neq 0$;

2) $D f_{\lambda}(x) \rightarrow 0$ as $x \rightarrow 0$;

3) Let $F: \Lambda \rightarrow V$ be defined by $F(\lambda)=\left(f_{\lambda}(0+), f_{\lambda}(0-)\right)$ then $F(\partial \Lambda) \subset \partial V$ and the degree of the map $F \mid \partial \Lambda$ is different from zero.

If $g \in \mathcal{L}^{r}, r \geq 0$, is a simple Lorenz map with finite critical orbits then there exists $\lambda$ and a maximal semi conjugation $h$ from $f_{\lambda}$ to $g$.

The proof of this Proposition is a corrected version of the proof of a similar statement for continous interval maps presented in [MS].

Proof. Let $g \in \mathcal{L}^{r}, r \geq 0$ be a simple Lorenz map with finite critical orbits.

Let $P(g)=\left\{z_{1}<\cdots<z_{l}=0<z_{l+1}<\cdots<z_{k}\right\}$ be the post-critical set of $g$ where $-1 \leq z_{1}=g\left(0_{+}\right) \leq 0$ and $0 \leq z_{k}=g\left(0_{-}\right) \leq 1$ are the critical values of $g$. The order of these points and the mapping $c:\{1,2, \ldots, l-1, l+1, \ldots k\} \rightarrow\{1, \ldots k\}$ defined by $g\left(z_{i}\right)=z_{c(i)}$ describes the combinatorics which we want to show can be realized by some map of our family. Consider the $k-1$ dimensional simplex $P=\left\{x \in R^{k} \mid-1=x_{0} \leq\right.$ $\left.x_{1} \leq \cdots \leq x_{l}=0 \leq x_{l+1} \leq \cdots \leq x_{k} \leq x_{k+1}=1\right\}$ and let $P_{0}$ be the interior of $P$. Let pr: $P \rightarrow V$ be the projection $\operatorname{pr}(x)=\left(x_{1}, x_{k}\right)$ and

$$
Z=\{(\lambda, x) \in \Lambda \times P \mid p r(x)=F(\lambda)\}
$$

Let us consider the mapping $T: Z \rightarrow P,(\lambda, x) \mapsto y$, where $f_{\lambda}\left(y_{i}\right)=x_{c(i)}$ and $y_{i}$ has the same sign as $x_{i}$. It is clear that $T$ is well defined and continuous. To finish the proof we have to find $(\lambda, x) \in Z$ such that $T(\lambda, x)=x$.

Let $\rho(x)=\min \left\{\left|x_{i}-x_{i+1}\right| ; i=0,1, \ldots, k\right\}$ and $d(x, y)=\max \left\{\left|x_{i}-y_{i}\right| ; i=1, \ldots, k\right\}$. We need the following:

Lemma 4.2. If $\left(\lambda_{n}, x(n)\right) \in Z$ is such that $\rho(x(n)) \rightarrow 0$ then

$$
\lim _{n \rightarrow \infty} \frac{d\left(T\left(\lambda_{n}, x(n)\right), x(n)\right)}{\rho(x(n))}=\infty
$$

Proof. Suppose, by contradiction, that there exists $K_{0}>0$ and a sequence $\left(\lambda_{n}, x(n)\right) \in Z$ such that $\rho(x(n)) \rightarrow 0$ and

$$
d(x(n), y(n)) \leq K_{0} \rho(x(n))
$$

where $y(n)=T\left(\lambda_{n}, x(n)\right)$. 
Claim 1. There exists constant $K_{1}>0$ such that if $x_{i}(n), x_{j}(n)$ have the same sign then

$$
\left|x_{c(i)}(n)-x_{c(j)}(n)\right| \leq K\left|x_{i}(n)-x_{j}(n)\right|+K_{1} \rho(x(n)) .
$$

Since $D f_{\lambda}(t) \leq K$, it follows from the Mean Value Theorem that

$$
\begin{aligned}
\left|x_{c(i)}(n)-x_{c(j)}(n)\right| & \leq K\left|y_{i}(n)-y_{j}(n)\right| \\
& \leq K\left|x_{i}(n)-x_{j}(n)\right|+K\left|y_{i}(n)-x_{i}(n)\right|+K\left|y_{j}(n)-x_{j}(n)\right| \\
& \leq K\left|x_{i}(n)-x_{j}(n)\right|+2 K_{0} \rho(x(n))
\end{aligned}
$$

and the claim is proved.

From Claim 1 we get by induction, for all $s$ there exists constant $C_{s}>0$ such that if $x_{c^{k}(i)}(n)$ and $x_{c^{k}(j)}(n)$ have the same sign for $k<s$ then,

$$
\left|x_{c^{s}(i)}(n)-x_{c^{s}(j)}(n)\right| \leq C_{s}\left|x_{i}(n)-x_{j}(n)\right|+C_{s} \rho(x(n))
$$

Claim 2. There exists $s_{0}$ such that for all $m \in\{1, \ldots, k\}$ there exists $s \leq s_{0}$ with $0 \in$ $\left[x_{c^{s}(m)}(n), x_{c^{s}(m+1)}(n)\right]$.

Consider the set $\mathcal{P}$ of pairs $\left(z_{i}, z_{j}\right)$ such that $0 \notin\left[z_{i}, z_{j}\right]$. If the claim is false then, since $\mathcal{P}$ is finite, there exists a periodic pair in $\mathcal{P}$. This implies the existence of a periodic homterval non-essential for $g$.

To finish the proof of the lemma let $m$ be such that $\rho(x(n))=\left|x_{m}(n)-x_{m+1}(n)\right|$ ( $m$ depends on $n)$. Let $s \leq s_{0}$ be such that $0 \in\left[x_{c^{s}(m)}(n), x_{c^{s}(m+1)}(n)\right]$ and $x_{c^{k}(m)}(n), x_{c^{k}(m+1)}(n)$ have the same sign for $k<s$.

From Claim 2 we get some $t$, depending on $n$ such that $\left|x_{t}(n)-x_{t+1}(n)\right| \leq C_{s} \rho(x(n))$ and either $x_{t}(n)=0$ or $x_{t+1}(n)=0$. Since $\left|f_{\lambda_{n}}\left(\left[y_{t}(n), y_{t+1}(n)\right]\right)\right|=\left|\left[x_{c(t)}(n), x_{c(t+1)}(n)\right]\right| \geq$ $\rho(x(n))$ we have that $D f_{\lambda_{n}}\left(\theta_{n}\right)\left|y_{t}(n)-y_{t+1}(n)\right| \geq \rho(x(n))$ for some $\theta_{n} \in\left[y_{t}(n), y_{t+1}(n)\right]$. Consider the case when $x_{t+1}(n)=0$. Then also $y_{t+1}(n)=0$. Therefore,

$$
\begin{aligned}
d(y(n), x(n)) & \geq\left|y_{t}(n)-x_{t}(n)\right| \\
& \geq \frac{1}{D f_{\lambda_{n}}\left(\theta_{n}\right)} \cdot \rho(x(n))-C \cdot \rho(x(n)) \\
& \geq\left(\frac{1}{D f_{\lambda_{n}}\left(\theta_{n}\right)}-C\right) \rho(x(n))
\end{aligned}
$$

On the other hand, $\left|y_{j}(n)-x_{j}(n)\right| \leq d(y(n), x(n)) \leq K_{0} \rho(x(n)) \rightarrow 0$ as $n \rightarrow \infty$. This implies that $D f_{\lambda_{n}}\left(\theta_{n}\right) \rightarrow 0$. This contradicts $d(x(n), y(n)) \leq K_{0} \rho(x(n))$.

(Lemma 4.2)

Assume that there is no fixed point for the mapping $T: Z \rightarrow P$. 
Lemma 4.3. There exists a continuous one parameter family of continuous mappings $G_{t}: Z \rightarrow P$ such that

1 ) $G_{0}(\lambda, x)=x$

2) $G_{t}(\lambda, x)=x$ if $x \in \partial P$

3) $G_{1}(Z) \subset \partial P$.

Proof. Let $D=\left\{x \in \mathbb{R}^{k-1} \mid \sum_{i=1}^{k-1} x_{i}^{2} \leq 1\right\}$ be the closed unit ball in $\mathbf{R}^{k-1}$ and let $\Phi: P \rightarrow D$ be a bi-Lipschitz homeomorphism. Let $\tilde{T}: Z \rightarrow D$ be the mapping $\tilde{T}=\Phi \circ T$. Since $\Phi$ is bi-Lipschitz and the distance from $x$ to the boundary of $P$ is equal to $\frac{1}{2} \rho(x)$ we have that, if $\left(\lambda_{n}, x(n)\right) \in Z$ is such that $\rho(x(n)) \rightarrow 0$ then

$$
\frac{d\left(\Phi\left(x_{n}\right), \tilde{T}\left(\lambda_{n}, x(n)\right)\right.}{d(\Phi(x(n)), \partial D)} \rightarrow \infty
$$

Now define $\tilde{G}_{1}(\lambda, x)$ as the point in the boundary of $D$ such that $\Phi(x)$ lies in the line segment bounded by $\tilde{T}(\lambda, x)$ and $\tilde{G}_{1}(\lambda, x)$. It follows that $\tilde{G}_{1}$ is continuous and $\tilde{G}_{1}(\lambda, x)=$ $\Phi(x)$ for $x \in \partial P$. Define $\tilde{G}_{t}=t \tilde{G}_{1}+(1-t) \Phi$ and $G_{t}=\Phi^{-1} \circ \tilde{G}_{t}$. This clearly satisfies the conditions of the Lemma.

(Lemma 4.3)

Lemma 4.4. Let $h: V \rightarrow \Lambda$ be a homeomorphism such that $\pi_{i}(x)=0 \Rightarrow \pi_{i}(F(h(x))=0$ for $i=1,2$, where $\pi_{i}: V \rightarrow \mathbf{R}$ is the projection in the $i$-th coordinate. Then there exists a mapping $H: P \rightarrow Z$ with the following properties:

1) $H$ is continuous and the restriction of $H$ to the interior of $P$ is a homeomorphism;

2) $\pi \circ H=h \circ p r$ where $\pi: Z \rightarrow \Lambda$ is the projection $(\lambda, x) \mapsto \lambda$;

3) the restriction of $H$ to each fiber $p^{-1}(v) \cup$ int $(P)$ is a diffeomorphism onto the fiber $\pi^{-1}(h(v)) \cup \operatorname{int}(Z)$.

Proof. Let $v=\left(v_{1}, v_{2}\right)$ and $F(h(v))=\left(\tilde{v_{1}}, \tilde{v_{2}}\right)$. Let $H_{v}: \mathbb{R} \rightarrow \mathbb{R}$ be the Moebius transformation that maps $v_{1}$ to $\tilde{v_{1}}, 0$ to 0 and $v_{2}$ to $\tilde{v}_{2}$. For $x \in p r^{-1}(v) \cap \operatorname{int}(P)$ define $H(x)=\left(h(v),\left(H_{v}\left(x_{1}\right), \cdots, H_{v}\left(x_{k}\right)\right)\right)$.

Choose a homeomorphsim $h: V \rightarrow \Lambda$ with the property needed to apply Lemma 4.4 and let $H: P \rightarrow Z$ be the corresponding map from Lemma 4.4. Let $\hat{G}_{t}=G_{t} \circ H: P \rightarrow P$. We have that

1) $\hat{G}_{t}$ is continuous and depends continuously on $t$;

2) $\hat{G}_{t}(\partial P) \subset \partial P$ and $\hat{G}_{t}(\operatorname{int}(P)) \subset \operatorname{int}(P)$;

3) $\hat{G}_{t}(x)=G_{0}(H(x))$ for all $x \in \partial P$;

4) $\hat{G}_{1}(P) \subset \partial P$;

5) The degree of $\hat{G}_{0}$ is equal to the degree of $F$.

The only statement that needs a proof is 5). Notice that

$$
p r \circ \hat{G}_{0}=(F \circ h) \circ p r
$$


and the restriction of $\hat{G}_{0}$ to $p r^{-1}(v) \cap \operatorname{int}(P)$ is a diffeomorphism onto $p r^{-1}(F(h(v)) \cap \operatorname{int}(P)$. Hence the degree of $\hat{G}_{0}$ is equal to the degree of $F \circ h$. Which is equal to the degree of $F$. Here we are using the following topological fact. Let $\Phi: D \rightarrow D$ be a continuous mapping that maps $\partial D$ onto $\partial D, \operatorname{int}(D)$ onto $\operatorname{int}(D)$ and $\left.\Phi\right|_{\operatorname{int}(D)}$ is smooth. Then the degree of $\left.\Phi\right|_{\partial D}$ equals the degree of $\Phi \mid \operatorname{int}(D)$, (see [D, p 67]). This is a contradiction.

It follows that there is a map $f_{\lambda}$ in the family which has also periodic critical orbits. Moreover, the combinatorics of these critical orbits are the same as the combinatorics of the critical orbits of $g$.

Let $h_{n}, n \geq 0$ be the homeomorphism that maps $B_{n}(f)$ into $B_{n}(g)$ given by Lemma 2.1. Because $g$ is a simple Lorenz map it follows that $h_{n}$ converges to a maximal semi conjugacy $h$ from $f$ to $g$.

(Proposition 4.1)

\section{Archipelagoes in the parameter plane}

In this section we will study the parameter plane of a given monotone Lorenz family $F:[0,1] \times[0,1] \rightarrow \mathcal{L}^{r}, r \geq 0$. The main object of our study is to understand the topological structure of Archipelagoes.

Let us concentrate on the archipelago $A=A_{\alpha, \beta}$. For every $\lambda \in A$ the domain of an $(\alpha, \beta)$-renormalization is denoted by $\left(p_{\lambda}, q_{\lambda}\right)$. Furthermore let $a=|\alpha|$ and $b=|\beta|$.

Proposition 5.1 (Island Structure). Let I be an island of the archipelago A. Then

1) For all $u \in U, M_{u} \cap A_{\alpha, \beta}=M_{u} \cap I$.

2) There exists an interval $\left[u_{1}, u_{2}\right] \subset U$ and Lipschitz functions $\partial_{+}, \partial_{-}:\left[u_{1}, u_{2}\right] \rightarrow M$ with Lipschitz constant 1 and $\partial_{-}(x)<\partial_{+}(x), x \in\left(u_{1}, u_{2}\right)$, such that

$$
I=\left\{(u, m) \mid u \in\left(u_{1}, u_{2}\right) \text { and } \partial_{-}(u)<m<\partial_{+}(u)\right\}
$$

Furthermore $\partial_{-}\left(u_{1}\right)=\partial_{+}\left(u_{1}\right)$ and $\partial_{-}\left(u_{2}\right)=\partial_{+}\left(u_{2}\right)$.

3) If $\lambda=\left(u_{1}, \partial_{-}\left(u_{1}\right)\right)$ or $\lambda=\left(u_{2}, \partial_{-}\left(u_{2}\right)\right)$, one of the extremal points of the island then

$$
f_{\lambda}^{a}\left(0_{-}\right)=0 \text { and } f_{\lambda}^{b}\left(0_{+}\right)=0
$$

or

$$
f_{\lambda}^{a}\left(0_{-}\right)=q_{\lambda} \text { and } f_{\lambda}^{b}\left(0_{+}\right)=p_{\lambda} .
$$

The first possibility is called a trivial extremal point, the second a full-branch extremal point.

4) For all $\lambda \in \partial_{+}$

$$
f_{\lambda}^{a}\left(0_{-}\right)=q_{\lambda} \text { or } f_{\lambda}^{b}\left(0_{+}\right)=0 .
$$

5) For all $\lambda \in \partial_{-}$

$$
f_{\lambda}^{a}\left(0_{-}\right)=0 \text { or } f_{\lambda}^{b}\left(0_{+}\right)=p_{\lambda} .
$$


proof. Let $\left(p_{m}, q_{m}, f_{m}^{a}, f_{m}^{b}\right)$ be the renormalization of $f_{m} \in I \cap M_{u}$. Observe that $m \mapsto q_{m}$ and $m \mapsto p_{m}$ are strictly monotone decreasing and $m \mapsto f_{m}^{a}\left(0_{-}\right)$and $m \mapsto f_{m}^{b}\left(0_{+}\right)$are strictly monotone increasing. This implies that once we arrive at the boundary of $I \cap M_{u}$, by increasing $m$ up to $m_{+}$we are in the situation

$$
f_{m}^{a}\left(0_{-}\right)=q_{m} \text { or } f_{m}^{b}\left(0_{+}\right)=0 .
$$

In particular for any $m>m_{+}$we have

$$
\theta^{-}\left(m^{\prime}\right)>\alpha \beta^{\infty} \text { or } \theta^{+}\left(m^{\prime}\right) \geq \beta^{\infty}
$$

and hence $m^{\prime} \notin A$. Similarly we study what happens when $m$ decreases up to the boundary of $I \cap M_{u}$. We proved that

$$
A \cap M_{u}=I \cap M_{u}
$$

and that this intersection is an interval $\left(\partial_{-}(u), \partial_{+}(u)\right.$. The boundary of $I \cap M_{u}$ consists of points $\partial_{-}(u)$ and points $\partial_{+}(u)$ : the boundary of $I$ consists of two parts, $\partial_{-} I$ and $\partial_{+} I$. Moreover

$$
f_{\partial_{-}(u)}^{b}\left(0_{+}\right)=p_{\partial_{-}(u)} \text { or } f_{\partial_{-}(u)}^{a}\left(0_{-}\right)=0
$$

and

$$
f_{\partial_{+}(u)}^{a}\left(0_{-}\right)=q_{\partial_{+}(u)} \text { or } f_{\partial_{+}(u)}^{b}\left(0_{+}\right)=0 .
$$

The island $I$ is open and connected. Hence the set of values $u$ for which $M_{u} \cap I \neq \emptyset$ is an open interval, say $\left(u_{1}, u_{2}\right)$. Now observe that for any $z \in \partial_{ \pm} I$ we have

$$
\partial_{ \pm} I \subset B_{z}
$$

where $B_{z}$ is the complement of the cones $C_{z}^{+}, C_{z}^{-}$defined in section 2 . In particular the functions $\partial_{ \pm}:\left(u_{1}, u_{2}\right) \rightarrow M$ are Lipschitz.

Left is to explain the behavior of the boundary points $\partial_{ \pm}(u)$ when $u$ tends to $u_{1}$ or $u_{2}$. For every $u \in\left(u_{1}, u_{2}\right)$ we have $\partial_{-}(u)<\partial_{+}(u)$. Let us assume that also $\partial_{-}\left(u_{1}\right)<\partial_{+}\left(u_{1}\right)$. The archipelago $A$ is closed. Hence for any $\left(u_{1}, m\right)$ with $\partial_{-}(u)<m<\partial_{+}(u)$ there is renormalization $\left(p_{m}, q_{m}, f_{m}^{a}, f_{m}^{b}\right)$. Because $\left(u_{1}, m\right) \in C_{-}\left(\left(u_{1}, \partial_{+}\left(u_{1}\right)\right)\right.$ and $\left(u_{1}, m\right) \in$ $C_{+}\left(\left(u_{1}, \partial_{-}\left(u_{1}\right)\right)\right.$ we have $f_{m}^{a}\left(0_{-}\right)<q_{m}$ and $f^{b}\left(0_{+}\right)>p_{m}$. Because of Lemma 3.1 the point $\left(u_{1}, m\right)$ is in the interior of $I$. This is a contradiction, proving that $\partial_{-}\left(u_{1}\right)=\partial_{+}\left(u_{1}\right)$.

(Proposition 5.1)

We have to distinguish special points on the boundary of islands. One type of special points are the extremal points, discussed in the above proposition. The other type of special points are vertices. Let $\partial_{-}$be the lower part of the boundary of the island $I$. A point $\lambda \in \partial_{-}$in the lower boundary is called a vertex if

$$
f_{\lambda}^{a}\left(0_{-}\right)=q_{\lambda} \text { and } f_{\lambda}^{b}\left(0_{+}\right)=0 .
$$

A vertex in the upper boundary is defined similarly. 
Lemma 5.2. Every archipelago contains only finitely many islands with a vertex.

proof. Suppose the archipelago $A_{\alpha, \beta}$ has infinitely many island $I_{n}$ which has a vertex $v_{n} \in \partial_{+}^{n}$. Here $\partial_{+}^{n}$ denotes the upper boundary of $I_{n}$. We may assume that $v_{n} \rightarrow v$. Since archipelagoes are closed sets, $v \in A_{\alpha, \beta}$.

The topological type of the maps in the vertices are all the same and it follows easily that the map corresponding to the parameter $v$ inherits this type also. So

$$
f_{v}^{a}\left(0_{-}\right)=q_{v} \text { and } f_{v}^{b}\left(0_{+}\right)=0 .
$$

By decreasing both parameters a little bit, we observe that $v$ is actually a vertex of some island $I$ in the same archipelago $A_{\alpha, \beta}$.

From Proposition 5.1 we get a neighborhood $(a, b) \times M$ of $I$ such that the archipelago intersects this neighborhood only in $I$, there are no other islands intersecting this neighborhood. But the vertex $v$ of $I$ lies in this neighborhood, it cannot be accumulated by the islands $I_{n}$. This is a contradiction.

(Lemma 5.2)

Lemma 5.3. Let $\lambda$ be a parameter for which $0_{-}$is periodic. Say with period $a=|\alpha|$, where $\alpha$ is the word describing the combinatorics of the orbit of $0_{-}$. Denote the set of parameters for which $0_{-}$is periodic with the same combinatorics $\alpha$ by $\alpha_{-}$.

Then there exists an interval $I \subset U$ and a Lipschitz function $\gamma: I \rightarrow M$ with Lipschitz constant 1 such that $I \times M$ is a neighborhood of $\lambda$ and

$$
\operatorname{graph}(\gamma) \cap(I \times M)=\alpha_{-} \cap(I \times M) .
$$

Proof. Let $z=(u, m) \in \alpha_{-}$. The monotonicity of the family implies

$$
\alpha_{-} \subset B_{z}
$$

and

$$
\begin{aligned}
& K^{-}\left(z^{\prime}\right)<\alpha, z^{\prime} \in \partial C_{z}^{-} \backslash\{z\} \\
& K^{-}\left(z^{\prime}\right)>\alpha, z^{\prime} \in \partial C_{z}^{+} \backslash\{z\} .
\end{aligned}
$$

For a small enough nieghborhood $I \times N$ of $z \in \alpha_{-}$the orbit of $0_{-}$will have the combinatorics given by $\alpha$ except for the last symbol. For each $u^{\prime} \in I$ let $m_{ \pm}^{\prime}$ be such that $\left(u^{\prime}, m_{ \pm}^{\prime}\right) \in$ $\partial C^{ \pm}(z)$. According to the above observation we have

$$
\begin{aligned}
& K^{-}\left(\left(u^{\prime}, m_{-}^{\prime}\right)\right)<\alpha \\
& K^{-}\left(\left(u^{\prime}, m_{+}^{\prime}\right)\right)>\alpha .
\end{aligned}
$$

In particular for each $u^{\prime} \in I$ there exists a unique $m^{\prime}\left(u^{\prime}\right) \in\left(m_{-}^{\prime}, m_{+}^{\prime}\right) \subset M_{u^{\prime}}$ with

$$
\left(u^{\prime}, m^{\prime}\left(u^{\prime}\right)\right) \in \alpha_{-}
$$

the set $\alpha_{-} \cap I \times M$ is the graph of the function $m^{\prime}: I \rightarrow M$. The first property discussed in this proof, namely $\alpha_{-} \subset B_{z}$ for each $z \in \alpha_{-}$implies that this function $m^{\prime}$ is 1 -Lipschitz. (Lemma 5.3) 
Lemma 5.4. Let $\lambda$ be a parameter for which the map $f_{\lambda}$ has two periodic points $p<0<q$. Say with period $a=|\alpha|$ and $b=\beta$ respectively. Furthermore assume

1) $(p, q) \cap(\operatorname{orb}(p) \cup \operatorname{orb}(q))=\emptyset$.

2) $f^{a} \mid[p, 0]$ and $f^{b} \mid[0, q]$ are monotone.

3) $D f^{a}(p)>1$ and $D f^{b}(q)>1$.

4) $f^{a}([p, 0])=[p, q]$

The word $\alpha \beta^{\infty}$ describes the combinatorics of the orbit of $0_{-}$. Denote the set of parameters for which the corresponding map has the properties 1), 2), 3) and 4) by $\alpha \beta_{-}^{\infty}$.

Then there exists an interval $I \subset U$ and a Lipschitz function $\gamma: I \rightarrow M$ with Lipschitz constant 1 such that $I \times M$ is a neighborhood of $\lambda$ and

$$
\operatorname{graph}(\gamma) \cap(I \times M)=\alpha \beta_{-}^{\infty} \cap(I \times M)
$$

Proof. Let $z \in \alpha \beta_{-}^{\infty}$. The properties 1), 2) and 3) hold in a neighborhood of $z$. The proof continues in this neighborhood as the proof of Lemma 5.3.

(Lemma 5.4)

Similar statements hold for the combinatorics of the orbit of $0_{+}$. The corresponding sets of parameters will be denoted by resp. $\beta_{+}$and $\beta \alpha_{+}^{\infty}$.

The uniqueness part of the above Lemma allow us to consider maximal arcs: the connected components of the sets $\alpha_{-}$and $\alpha \beta_{-}^{\infty}$ are graphs of Lipschitz functions.

Lemma 5.5. Let $\gamma:\left(t_{0}, t_{1}\right) \rightarrow M$ be the Lipschitz function whose graph is a component of $\alpha \beta_{-}^{\infty}$. Then

1) This function can be extended to a Lipschitz function on $\left[t_{0}, t_{1}\right]$.

2) There exists a function $\gamma_{2}:\left[t_{0}, t_{1}\right] \rightarrow M$ whose graph is contained in $\beta_{+}$.

3) If there exits $(u, m) \in\left(\operatorname{graph}(\gamma) \cap A_{\alpha, \beta}\right) \backslash \beta_{+}$then $\beta_{+}$intersects each component of $\operatorname{graph}(\gamma) \backslash\{\lambda\}$ unless such a component terminates in the boundary of the parameter domain. Moreover $\gamma_{2}(u)>\gamma(u)$, the $\beta_{+}$curve lies above the point $(u, m)$ in the $\alpha \beta_{-}^{\infty}$ curve.

In particular a component of $\alpha \beta_{-}^{\infty} \backslash\{(u, m)\}$ which does not terminate in the boundary of the parameter domain contains a vertex in the upper boundary of an island of the archipelago $A_{\alpha, \beta}$.

Proof. Remember that the arc $\gamma:\left(t_{1}, t_{2}\right) \rightarrow M$ satisfies

$$
\operatorname{graph}(\gamma) \subset B_{(t, \gamma(t))}
$$

for all $t \in\left(t_{1}, t_{2}\right), \gamma$ is 1 -Lipschitz. Observe that the length of the interval $T_{t}=M_{t_{1}} \cap$ $B_{(t, \gamma(t))}$ tends to zero, when $t \rightarrow t_{1}$. Moreover these intervals $T_{t}$ with $t>t_{1}$ are nested: $T_{s} \subset T_{t}$ whenever $t_{1}<s<t$. The intersection of the intervals $T_{t}$ defines the continuous extension of the arc $\gamma$.

To proof 2) it is enough to show that $\beta_{+} \cap M_{t} \neq \emptyset$ for each $t \in\left(t_{1}, t_{2}\right)$. Let $t \in\left(t_{1}, t_{2}\right)$ and $\{\lambda\}=\alpha \beta_{-}^{\infty} \cap M_{t}$. 
case $I: f^{b}\left(0_{+}\right)>0$. Consider the maps in $M_{t}$ with the properties

1) there exists a branch $T=(0, x)$ of type $\beta$,

2) there exists $x_{0} \in T$ with $f^{b}\left(x_{0}\right)<x_{0}$,

3) $f^{b}\left(0_{+}\right)>0$.

Observe that property 2) and 3) imply the existence of a periodic attractor in $T$ which attracts the orbit of $0_{+}$. In particular, the orbit of $0_{+}$is infinite. This implies that $0 \notin \partial_{-} f^{i}(T)$ for each $i \leq b$. Consequently, if $(t, m)$ is a point with this properties there is an small interval $\{t\} \times(m-\epsilon, m]$ of maps with the three property.

Observe that the map in $\lambda$ has the three properties. Let $H=\{t\} \times\left(m_{1}, m_{0}\right]$ be the maximal interval of maps with the above properties. We are going to show that in $\left(t, m_{1}\right)$ the map is in $\beta_{+}$.

Observe that by decreasing $m \in\left(m_{1}, m_{0}\right]$ we see that the interval $T$ is increasing in length. Because the family is monotone, the same point $x_{0}$ in $T$ which was moved to the left persists to be in $T$ and will be moved to the left. As before we see that the orbit of the interval $T$ will never hit $0_{+}$.

We showed that property 1 ) and 2 ) hold also in $\left(t, m_{1}\right)$. Hence, in the boundary of $H$ property 3) has to be violated. Otherwise we could decrease $m$ slightly more.

case II: $f^{b}\left(0_{+}\right)<0$. Consider the maps in $M_{t}$ with the properties

1) there exists a branch $T=(0, x)$ of type $\beta$,

2) $f^{b}(x)>x$

3) $f^{b}\left(0_{+}\right)<0$.

Properties 2) and 3) imply that there is a hyperbolic periodic point $q \in T$ of type $\beta$. Consequently, the branch of type $\beta$ will persist under small perturbations. The collection of above maps is open.

Observe that the map in $\lambda$ satisfies the above properties. We have $f^{b}(x)>x$ otherwise the orbit of $0_{-}$could not be trapped in the orbit of type $\beta$, which is the case in $\lambda \in \alpha \beta_{-}^{\infty}$. Let $H=\{t\} \times\left[m_{0}, m_{1}\right)$ be the maximal interval of maps with the above properties. We are going to prove that $\left(t, m_{1}\right) \in \beta_{+}$.

Observe that by increasing $m \in\left[m_{0}, m_{1}\right)$ the branch $T$ will be decreasing, a consequence of the fact that the family is monotone. However the periodic orbit persists and there has to be a periodic orbit at $\left(t, m_{1}\right)$. Maybe not hyperbolic anymore. In particular, there is a branch $T=(0, x)$ of type $\beta$ in $\left(t, m_{1}\right)$.

Again from the monotonicity of the family we get that $f^{b}(x)>x$ in $\left(t, m_{1}\right)$. The only way to reach the boundary of $H$ is to violate property 3$):\left(t, m_{1}\right) \in \beta_{+}$.

To prove 3) Let $\left.(t, m)=\lambda \in \operatorname{graph}(\gamma) \cap A_{\alpha, \beta}\right) \backslash \beta_{+}$. The $M_{t}$ contains a point in $\beta_{+}$. Because $(t, m) \in A_{\alpha, \beta} \backslash \beta_{+}$, there is a renormalization, we get that $\gamma_{2}(t)>\gamma(t)$. To finish the proof of 3$)$ we have to show that $f^{b}\left(0_{+}\right)>0$ in $\left(t_{1}, \gamma\left(t_{1}\right)\right.$.

Because $\left(t_{1}, \gamma\left(t_{1}\right)\right)$ is a boundary point we get that $D f^{b}(q)=1$. Otherwise we could extend $\alpha \beta_{-}^{\infty}$. The map under consideration has negative Schwarzian derivative. Hence, 
the neutral periodic point attracts a critical point with an infinite orbit. The orbit of $0_{-}$ is trapped in the orbit of $q$. Only the orbit of $0_{+}$can be attracted towards $q$. In particular $f^{b}\left(0_{+}\right)>0$.

A similar Lemma holds for the combinatorics of $0_{+}$. In the next Lemma we will see that the symmetry breaks down. Moving up or down has well understood consequences on the combinatorics of the map. On the other hand moving one branch up and the other down are the deformations which are difficult to understand, the deformations in directions parallel to U. The next Lemma indicates a difference between such movements to the right and to the left.

Lemma 5.6. Let $\lambda \in \alpha \beta_{-}^{\infty} \cap \beta \alpha_{+}^{\infty}$, say $\lambda=(u, m)$. Then there exists a parameter $\mu=\left(t, m^{\prime}\right)$ such that

1) $t \leq u$.

2) $\mu$ is the right extremal point of an island $I$ of the archipelago $A_{\alpha, \beta}$. Moreover it is a full branch extremal point.

3) The island I has a vertex in its upper (and lower) boundary.

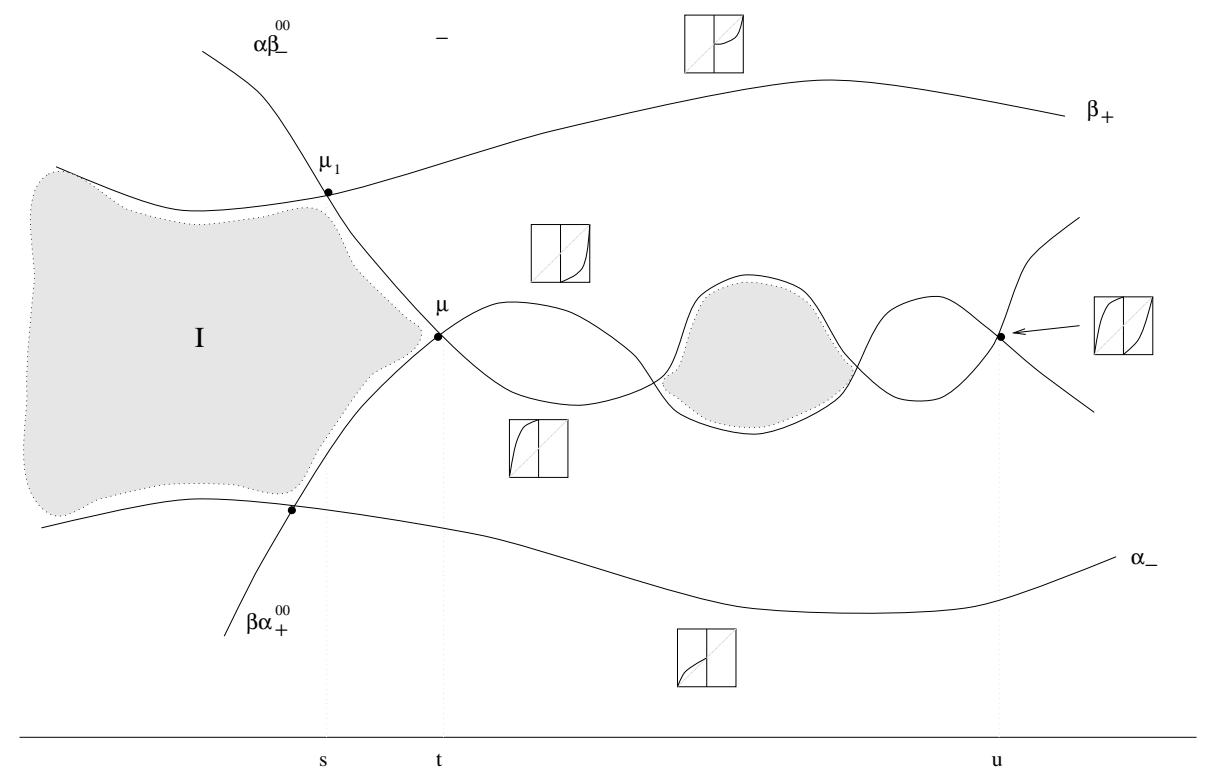

Figure 2 Illustration to the proof of Lemma 5.6

proof. Let $\gamma_{+}:\left(t_{+}, u\right] \rightarrow M$ be the function whose graph is the component of $\alpha \beta_{-}^{\infty} \backslash\{\lambda\}$ which touches $\lambda$ from the left side. Similarly $\gamma_{-}:\left(t_{-}, u\right] \rightarrow M$ be the function whose graph is the component of $\beta \alpha_{+}^{\infty} \backslash\{\lambda\}$ which also touches $\lambda$ from the left side.

Let $t \in\left[t_{+}, u\right] \cap\left[t_{-}, u\right]$ be minimal such that $\gamma_{+}(t)=\gamma_{-}(t)$, describing the left most intersection point of $\alpha \beta_{-}^{\infty}$ with $\beta \alpha_{+}^{\infty}$. Let $\mu$ be the intersection point. Observe that Lemma 5.4 can be applied in this point, giving us local extensions of the $\operatorname{arcs} \alpha \beta_{-}^{\infty}$ and $\beta \alpha_{+}^{\infty}$. So $\mu$ is a point in the interior of both arcs. 
Actually $\mu \in A_{\alpha, \beta}$, the map is of full branch type. So we can apply Lemma 5.5: let $s \leq t$ be maximal such that $\mu_{1}=\left(s, \gamma_{+}(s)\right) \in \beta_{+} \cap \alpha \beta_{-}^{\infty}$. Observe that $\alpha \beta_{-}^{\infty}$ can not terminate to the left in the boundary of parameter space.

Clearly $\mu_{1}$ is a vertex point in the upper boundary of some island $I \subset A_{\alpha, \beta}$. Observe that the island $I$ intersects $[s, t] \times M$ only below the graph of $\gamma_{+} \mid[s, t]$, moving up a point in this graph would destroy the renormalization immediately.

The aim is to show that the graph of $\gamma_{+} \mid[s, t]$ is part of the upper boundary of the island $I$. From Lemma 5.5 we get that the arc $\beta_{+}$lies above the point $\mu$. Furthermore $\mu_{1}$ is the right most intersection of the graph of $\gamma_{+} \mid\left(t_{+}, t\right]$ with the $\beta_{+}$arc. So the $\beta_{+}$arc in the strip $(s, t) \times M$ lies above the graph of $\gamma_{+}$, which lies above the island $I$. So the boundary points of the island $I$ in the strip $(s, t) \times M$ lie in the graph of $\gamma_{+}$, boundary points in the upper boundary can only be of two types $\alpha \beta^{\infty}$ or $\beta_{+}$.

Left is to show that the boundary actually extends up to $\mu$. Clearly the piece of the boundary in the strip can not have vertices anymore, for this you need points in $\beta_{+}$. So the graph contains the right extremal point of the island. This extremal point can not be trivial, for this you need a point in $\beta_{+}$. So it is of full branch type. The only parameter of this type in the graph of $\gamma_{+} \mid[s, t]$ is $\mu$. Clearly the island does not extend beyond $\mu$, the archipelago intersects $\{t\} \times M$ only in $\mu$.

(Lemma 5.6)

\section{Proof of Full-Island Theorem $\mathbf{1 . 9}$}

Fix a monotone Lorenz family $F:[0,1] \times[0,1] \rightarrow \mathcal{L}^{r}, r \geq 3$. Let $A=A_{\alpha, \beta} \subset[0,1] \times[0,1]$ be an the $\alpha, \beta$-archipelago.

Proposition 6.1. The archipelago $A$ has an island $I \subset A$ which has a trivial and a full branch extremal point.

proof. By Proposition 4.1 there exists $\lambda \in \alpha \beta_{-}^{\infty} \cap \beta \alpha_{+}^{\infty}$. Now Lemma 5.6 gives an island which has a vertex and its right extremal point is of full branch type. Because there are only finitely many islands with a vertex we can take the left most island $I \subset A_{\alpha, \beta}$ which has a vertex and whose right extremal point is of full branch type.

Left is to show that the left extremal point of this island is trivial. Suppose not and apply Lemma 5.6 again: we will find an island left of $I$ which has a vertex and whose right extremal point is of full branch type. But $I$ was the left most island with these properties. This is a contradiction.

(Proposition 6.1)

Lemma 6.2. For each Lorenz map $f \in \mathcal{L}^{2}$ there exists a simple Lorenz map $\hat{f}$ and a maximal semi conjugation from $f$ to $\hat{f}$.

Proof. Define $x \sim y$ if the there exists a countable closed set $C \subset[x, y]$ such that each connected component of $[x, y] \backslash C$ is a homterval. Clearly, it follows that $\sim$ is an equivalence relation and the equivalence classes are either points or closed intervals. Also $f$ maps 
equivalence classes into equivalence classes: if $[x]$ is the equivalence class of $x$ and $0 \notin \operatorname{int}[x]$ then

$$
f([x]) \subset[f(x)] .
$$

Therefore, the quotient space $[P, Q] / \sim$ is an interval and $f$ induces a Lorenz map on this interval which does not have homtervals.

The semi conjugacy being the quotient map $h$. Left is to show that $h$ is a maximal semi conjugacy. Suppose there exists $y \in[-1,1]$ such that $h^{-1}(y)$ is not a point neither a homterval. In this case $T=h^{-1}(y)$ contains a closed countable set $C$ such that the connected components of $T \backslash C$ are all homtervals. Because $C$ is countable and closed it has an isolated point $c \in C$. In particular there are two homtervals $I_{1}, I_{2} \subset T$ with $c$ as common boundary point. Clearly, this common boundary point is a preimage of 0 , say $f^{n}(c)=0$. In particular $f$ has two critical homtervals $f^{n}\left(I_{1}\right)$ and $f^{n}\left(I_{2}\right)$.

A consequence of these two critical homtervals and the fact that $f$ is $C^{2}$ is that $f$ does not have wandering intervals. If $f$ would have a wandering interval then we could modify the map on $f^{n}\left(I_{1}\right) \cup f^{n}\left(I_{2}\right)$ to obtain a smooth bimodal map with a wandering interval. In [MMS] this is proved to be impossible.

Every homterval of $f$ eventually falls into a periodic homterval. We proved that $h$ is a maximal semi conjugacy.

(Lemma 6.2)

Let us sumarize the possibilities in the above Lemma.

1) If $f$ has at most one critical homterval then $h^{-1}(y)$ is a point or a homterval for each $y \in[-1,1]$.

2) If $f$ has two critical homtervals, say $L$ and $R$ then

2a) The two intervals $L$ and $R$ are periodic with distinct orbits or

$2 \mathrm{~b})$ The two intervals are periodic with the same orbit or

2c) One interval is periodic and the other is eventually periodic or

2d) The two intervals are eventually periodic.

The notion of maximal semi conjugacy is needed to collapse al these periodic and eventually periodic homtervals. If the map $f$ would have at most one critical homterval the usual equivalence relation $x \sim y$ if an only iff $[x, y]$ is a homterval would give the maximal semi conjugacy to a simple Lorenz map.

Theorem 1.9. Let $I \subset A_{\alpha, \beta}$ be an island which has a trivial and a full branch extremal point. The Lorenz Family $G: I \rightarrow \mathcal{L}^{r}$ defined by

$$
I \ni \lambda \mapsto\left(p_{\lambda}, q_{\lambda}, f_{\lambda}^{a}, f_{\lambda}^{b}\right) \in \mathcal{L}^{r}
$$

is a full family.

Proof. Let $f$ be a Lorenz map. We would like to find a map $g=G(\lambda)$ which is essentially conjugated to $f$. By Lemma 6.2 we get a simple Lorenz map $\hat{f}$ and a maximal semi conjugation $h_{1}$ from $f$ to $\hat{f}$. 
Let $K_{n}^{ \pm}=K_{n}^{ \pm}(\hat{f}), n \geq 0$. Proposition 4.1 can be applied to the family $G$. Hence for each $n \geq 0$ there exists $\lambda_{n} \in I$ such that $K_{n}^{ \pm}=K_{n}^{ \pm}\left(g_{n}\right)$, where $g_{n}=G\left(\lambda_{n}\right)$. By Lemma 2.1 we get homeomorphisms $h_{n}:[-1,1] \rightarrow[-1,1]$ preserving the combinatorics up to time $n$ :

$$
h_{n}: \mathbb{B}_{n}\left(g_{n}\right) \rightarrow \mathbb{B}_{n}(\hat{f}) .
$$

We may assume that a subsequnce of $g_{n}$ converges to $g=G(\lambda)$. Then a continuity argument implies that $h_{n} \rightarrow h$, where $h:[-1,1] \rightarrow[-1,1]$ is a monotone increasing continuous map preserving combinatorics

$$
h: \mathbb{B}_{n}(g) \rightarrow \mathbb{B}_{n}(\hat{f}) .
$$

The partitions $\mathbb{B}_{n}(\hat{f})$ will get finer and finer. This is a consequence of the fact that $\hat{f}$ does not have homtervals. The map $h$ is continuous. It can happen that $g$ has homtervals. In this case the partitions $\mathbb{B}_{n}(g)$ will not get finer and finer. As consequence the map $h$ will have intervals which are mapped to points, $h$ is a semiconjugacy from $g$ to $\hat{f}$. We showed that $f$ and $g$ are essentially conjugated.

(Theorem 1.9)

\section{References}

[ACT] A. Arneodo, P. Coullet, C. Tresser, A possible new mechanism for the onset of turbulence, Physics Letters, 81A n. 4 (1981),197-201.

[D] A.Dold, Lectures on Algebraic Topology, Springer Verlag 1972.

[GW] J. Guckenheimer, R. F. Williams, Structure Stability of Lorenz attractors, Publ. Math. IHES (1979), 59-72.

[HS] J. H. Hubbard, C. T. Sparrow, The Classification of Topologically Expansive Lorenz Maps, Comm. on Pure and App. Math., XLIII, (1990), 431-443.

[L] E. N. Lorenz, Deterministic non-periodic flow. J. Atmos. Sci. 20 (1963), 130-141.

[M] R.Mañé, Hyperbolicity, Sinks and Measure in One-dimensional Dynamics Commun. Math. Phys. 100 (1985), 495-524 and Erratum Commun. Math. Phys. 112 (1987), 721-724.

[MMMS] M.Martens, W.de Melo, P.Mendes, S.van Strien On Cherry Flows, Erg.Th. \& Dyn.Sys. 10 (1990), 531-554.

[MMS] M. Martens, W. de Melo, S. van Strien, Julia-Fatou-Sullivan Theory for real one-dimensional dynamics, Acta Math. 168 (1992) 273-318.

[MS] W.de Melo, S.van Strien, One-dimensional Dynamics, Springer Verlag.

[MT] J. Milnor, W. Thurston, On iterated maps of the interval, Springer Lecture Notes in Mathematics 1342 (1988) 465-563.

[MP] W.de Melo, C.Pugh, On the $C^{1}$ Brunovski Hypothesis, J.Diff. Equations 113 (1994) 300-337. 
[P] W. Parry, Symbolic dynamics and transformations of the unit interval. Trans. Amer. Math. Soc. 122 (1966), 368-378.

[R] A.Rovella, The dynamics of perturbations of contracting Lorenz Maps, Bul.Soc.Brazil. Mat. (N.S.) 24 (1993) no.2, 233-259. 